\title{
Long terminal repeats (LTR) and transcription factors regulate $P H R E 1$ and PHRE2 activity in Moso bamboo under heat stress
}

\author{
Pradeep K. Papolu', Muthusamy Ramakrishnan ${ }^{1,2,3}$, Qiang Wei ${ }^{2,3}$, Kunnummal Kurungara Vinod ${ }^{4}$, \\ Long-Hai Zou ${ }^{1}$, Kim Yrjala', Ruslan Kalendar ${ }^{5}$ and Mingbing Zhou ${ }^{1,6^{*}}$
}

\begin{abstract}
Background: LTR retrotransposons play a significant role in plant growth, genome evolution, and environmental stress response, but their regulatory response to heat stress remains unclear. We have investigated the activities of two LTR retrotransposons, PHRE1 and PHRE2, of moso bamboo (Phyllostachys edulis) in response to heat stress.

Results: The differential overexpression of PHRE1 and PHRE2 with or without CaMV35s promoter showed enhanced expression under heat stress in transgenic plants. The transcriptional activity studies showed an increase in transposition activity and copy number among moso bamboo wild type and Arabidopsis transgenic plants under heat stress. Comparison of promoter activity in transgenic plants indicated that $5^{\prime}$ LTR promoter activity was higher than CaMV35s promoter. Additionally, yeast one-hybrid $(\mathrm{Y} 1 \mathrm{H})$ system and in planta biomolecular fluorescence complementation (BiFC) assay revealed interactions of heat-dependent transcription factors (TFs) with 5'LTR sequence and direct interactions of TFs with pol and gag.
\end{abstract}

Conclusions: Our results conclude that the $5^{\prime}$ LTR acts as a promoter and could regulate the LTR retrotransposons in moso bamboo under heat stress.

Keywords: Moso bamboo, LTR-retrotransposons, Transposable elements, PHRE1 and PHRE2, siRNA, Heat stress, Transposition

\section{Background}

Abundantly present in plant genomes, long terminal repeat (LTR) retrotransposons, class I transposable elements (TE), are characterized by a pair of identical repeats several hundred base pairs long on both the terminals. They belong to two major superfamilies, Ty1/Copia, and Ty3/Gypsy with pan-genome distribution and constitute a major portion of genomes [1,2]. The most common internal coding genes flanked by the LTRs are gag (group-associated antigene or group-specific

\footnotetext{
*Correspondence: zhoumingbing@zafu.edu.cn

${ }^{1}$ State Key Laboratory of Subtropical Silviculture, Zhejiang A\&F University, Lin'an, Hangzhou 311300, Zhejiang, China

Full list of author information is available at the end of the article
}

antigen) and pol (polymerase), involved in RNA synthesis. The pol is involved in reverse transcription and encodes pepsin-like aspartate proteases (PR), integrase (INT), reverse transcriptase (RT), and ribonuclease $\mathrm{H}$ $(\mathrm{RH})$ proteins. The $g a g$ is involved in the maturation, packaging of RNA-mediated RTs, and re-integration into the genome with the help of INT. LTR retrotransposons have other structural features such as primer binding site (PBS) and a poly-purine tract (PPT), necessary for reverse transcription and transposition $[3,4]$. The transcriptional activity of LTR retrotransposons is regulated by promoter elements in the 5' LTR and untranslated (UTR) regions. Due to their 'copy and paste' transposition activity LTR retrotransposons can generate new 
mutations within the genome $[4,5]$, including copy insertion, gene silencing, chromosomal rearrangements, and genome amplification. Although LTR retrotransposon mutations occur at random in an organism, only the most beneficial mutations are carried forward [6, 7].

Natural triggers of transposition activity of LTR retrotransposons remain unclear, but it is well documented that they can be activated by stress instances [8], and bear some epigenetic marks. In tobacco, the specific expression of the retroelements, Tnt1 and Tto 1 situated within the U3 region of 5'LTR fused by the GUS reporter gene have revealed transcriptional activity under the stress conditions [9-11]. Integrated into the genome of Arabidopsis and rice, Tnt1 and Tto1 attained transposition under stress conditions [12, 13]. Similarly, in oat (Avena sativa), a Ty1/Copia retrotransposon OARE1 was detected highly expressed under biotic and abiotic stresses [14], thus stimulating plant defense responses. A retrotransposon in Citrus limon, CLCoy1 was induced by salt and wounding stresses [15]. A recent study suggests that heat-activated Copia superfamily LTR retrotransposons in Arabidopsis increased nuclear size and strengthened chromatin reorganization [16]. Compared to normal conditions, GBRE-1 elements in Gossypium barbadense and G. hirsutum showed higher expression levels under heat stress [17]. HuTy1P4 retroelement in the pitaya (Hylocereus undatus) genome is transcriptionally activated by different stress conditions [18]. ONSEN, an element of Ty1/Copia superfamily, in Arabidopsis progeny lines was found to be most effective under heat stress [19].

Despite being significant components of the plant genome, most LTR retrotransposons remain silent because of the suppression by DNA and histone proteins modifications, recombination, and small RNAs [20-22]. With the presence of trans-acting small interfering RNAs (siRNAs), usually produced in high copy numbers, LTR retrotransposons are involved in gene regulation at the transcriptional and post-transcriptional levels [20, 23-25]. Heat stress adaptation in Arabidopsis is reported to activate a Ty1/Copia retrotransposon named ONSEN, which was found activated in mutants impaired in the biogenesis of siRNAs $[26,27]$. The transcriptional activation of ONSEN was regulated by the siRNA-related pathway and showed transgenerational transposition of the retroelement under heat stress [28]. Evidence for post-transcriptional and epigenetic control of Gypsy retroelements through regulation of piwi-interacting RNA (piRNA) have been reported in Drosophila, in which a heatresponsive stress chaperone heat shock protein 70 (HSP70) was found inducing the transposon activity [29]. The experience from plants so far indicates that
LTR retrotransposons functions are species-specific and are associated with environmental adaptation and provides an evolutionary advantage [30-32].

Among the bamboos, moso bamboo, Phyllostachys edulis (subfamily Bambusoideae) stands prominent among the 500 species belonging to 48 genera [33, 34] and is recognized for its economic use. It is acclaimed as one of the fastest-growing plants on earth, with a growth rate of 30 to $100 \mathrm{~cm}$ per day [35, 36]. Although grown in a wide range of climates, moso bamboo is typically temperate adapted and shows a long vegetative phase (usually 60 years) and monocarpic. The switch from a vegetative to a reproductive phase is unpredictable [37, 38]. Controlled cross-breeding and development of breeding lines and mapping populations are difficult in moso bamboo, and therefore, in genetic studies, it lags far behind other cultivated cereals [36]. The moso bamboo is large with a size closer to $2.0 \mathrm{Gbp}$ but is smaller than the maize genome [39]. Transposons occupy over $63.24 \%$ of the moso bamboo genome [40] consisting of $45.67 \%$ of retroelements. Among the retroelements, LTR retrotransposons occupy about 878 Mbp (43.89\%), a size equivalent to 2.25 times that of the rice genome [41-46].

In our previous studies, we have reported two LTR retrotransposons PHRE1 and PHRE2 (synonymized with $p h$-LTR1 and $p h$-LTR2, respectively) in the moso bamboo genome $[47,48]$. Selected based on the homology and structure with that of LTR retrotransposons, PHRE1 and PHRE2 contain the RT, RH, INT genes, PBS, and PPT, qualifying them to be capable of transposition. The open reading frames of these protein domains were complete and had no distinct mutations. The terminal repeats of $5^{\prime}$ and $3^{\prime}$ sequences of PHRE1 (98.5\%) and PHRE2 (98.3\%) share significant sequence homology. Zhou et al. [47, 48] found that under irradiation treatment, PHRE2 copy number increased in moso bamboo seedlings as well as in transgenic Arabidopsis plants. In Arabidopsis, however, higher transposition activity could be noticed T3 plants than in the T2 plants, and a detailed molecular and functional characterization has not been attempted in these studies $[47,48]$, analyzing the information on the native retrotransposon functionality. Thus, we have carried out this study to characterize molecular functions of PHRE1 and PHRE2 to fathom the basic retrotransposon functions as well as the promotor-mediated epigenetic regulation in response to heat stress. For characterization, we used the modified carbon nanotube diffusion method for moso bamboo transformation [49] and Agrobacterium floral dip method for Arabidopsis transformation to overexpress these elements. 


\section{Results}

\section{Structure analysis of PHRE1 and PHRE2}

PHRE1 and PHRE2 were selected based on the complete structure and homology of domains, complete with two full-length $g a g$ and pol genes with continuous ORFs without nonsense and frameshift mutations (Table S1). Both were Ty3/Gypsy LTR retrotransposons with 4980 bp (PHRE1) and 5515 bp (PHRE2) length $[47,48]$. The 5'LTR of PHRE1 contained core promoter features such as four CAAT boxes located at 39, 142, 144, and 291 bp positions and TATA box located at 49 bp positions. Two methyl jasmonate regulatory elements of TGACGmotif were located at 20 and 311 bp positions, and a GTGGC-motif was located at $10 \mathrm{bp}$ position. Three drought-responsive myeloblastosis (MYB) binding sites (MBS) were located at 16, 104, and $219 \mathrm{bp}$ positions, and a light-responsive MYB-recognition element (MRE) site is located at $215 \mathrm{bp}$ positions. In the PHRE2 element, the 5' LTR (670 bp) and 3'LTR (465 bp) of shared significant homology sequences (98.3\%). The core promoters, TATA box, and CAAT box were located at 280 and 267 bp positions, respectively. Additionally, PHRE2 had a salicylic acid (SA) related element at $193 \mathrm{bp}$, gibberellin (GA) related element at $94 \mathrm{bp}$, and temperature-responsive elements at $212 \mathrm{bp}$ and $133 \mathrm{bp}$ positions. The promoter also had cis-regulatory elements, such as methyl jasmonic acid (CCTGCA), auxin regulatory (TGA), drought-responsive (MYB), and abscisic acid (ABA) regulatory elements located at 193, 94, 212, and $133 \mathrm{bp}$ respectively $[47,48]$.

\section{Development of transgenic plants}

At least 40 Arabidopsis primary transformants (T0) of each PHRE1 and PHRE2 were generated using floral dip transformation. After hygromycin selection, the plants were established in a growth chamber where they developed normally and set seeds. Screening of putative transformants in $\mathrm{T} 1$ generation in the presence of $30 \mathrm{mg} / \mathrm{L}$ hygromycin resulted in survival (exhibited proper flowering, shoot, and root formation) of at least 20-25 independent events for each PHRE1 and PHRE2 (Fig S1). After 45 days, T1 plants were phenotypically and morphologically (flowering, shoot, root, plant height, and seed setting) similar to untransformed control plants grown under a non-selective medium. This indicated that neither the antibiotic resistance gene nor the LTR retrotransposons constructs had affected the growth of transformed plants. PCR analysis using primers specific for PHRE1, PHRE2, GUS, and antibiotic genes, confirmed the presence of T-DNA in the putative transformants (Fig. S2). Likewise, at least 20 moso bamboo transgenic plants were developed using carbon nanotubes transformation after 3 days post-infiltration. Reporter gene (GFP) expression was observed in moso bamboo transgenic plants by confocal microscopy imaging and performed qRT-PCR assay for the transformants.

\section{PHRE1 and PHRE2 show promoter activity in moso bamboo} Observed under a confocal microscope, after the GUS assay incubation for $72 \mathrm{~h}$, the mature leaves of the transgenic moso bamboo plants indicated differential expression for the presence of promoters (Fig. 1 and 2). While no GFP and GUS expressions were observed in the system driven by the CaMV35s promoter, the systems are driven by PHRE1 and PHRE2 showed clear expression patterns with bright green fluorescence with GPF and blue color of GUS in the leaves (Fig.1 and 2). No fluorescent expression was detected in negative controls, including delivery of free plasmid DNA, DNA-PEI without SWNTs, and PEI-SWNTs without plasmid DNA (Fig.1). This indicated that the transcriptions of these reporter genes were driven by the promoter of PHRE1 and PHRE2. In the transgenic Arabidopsis too, intense GUS staining was observed in the plants expressing the pMDC164:PHRE1 and pMDC164:PHRE2 (Fig. 3A and C) than pMDC43:PHRE1 and, pMDC43:PHRE2 (Fig. 3B and D). Notably, GUS transgene expression was absent in untransformed control plants. This implied that both PHRE1 and PHRE2 are active elements and have promoter activity.

The qRT-PCR on the mRNA from transgenic bamboo plants indicated that expression levels of 5' and 3' LTRs of PHRE1 were down-regulated by 2.56 - and 2.29- fold in the leaf, respectively, followed by the downregulation of $g a g$ and pol genes (1.93- and 1.90- fold changes) (Fig. S3 A). Similarly, 5' and 3' LTRs expression were downregulated by 2.44- and 2.39- folds in the leaf of PHRE2, respectively, followed by the downregulation of gag and pol transcripts (1.95- and 1.96-fold changes) (Fig. S3 A). A similar expression was not observed in the transgenic bamboo with pMDC43:PHRE1/PHRE2 having CaMV35s promoter. (Fig. S3 B). Also, in Arabidopsis, no transcripts corresponding to either PHRE1 or PHRE2 were detected in untransformed control plants. The gene expression data for the transgenic lines were presented relative to the Arabidopsis actin normalizer gene, and greater $\Delta \mathrm{Ct}$ values were obtained (difference between $\mathrm{Ct}$ mean of PHRE1/PHRE2 and Actin) in PHRE1 and PHRE2 lines with CaMV35s promoter, than for PHRE1 and PHRE2 (without CaMV35s promoter). This indicated a lower quantitative expression of 5'LTR, gag, pol, and 3'LTR in the root, leaf, and stem of PHRE1 and PHRE2 with CaMV35s promoter (Fig. S4 and S5). 


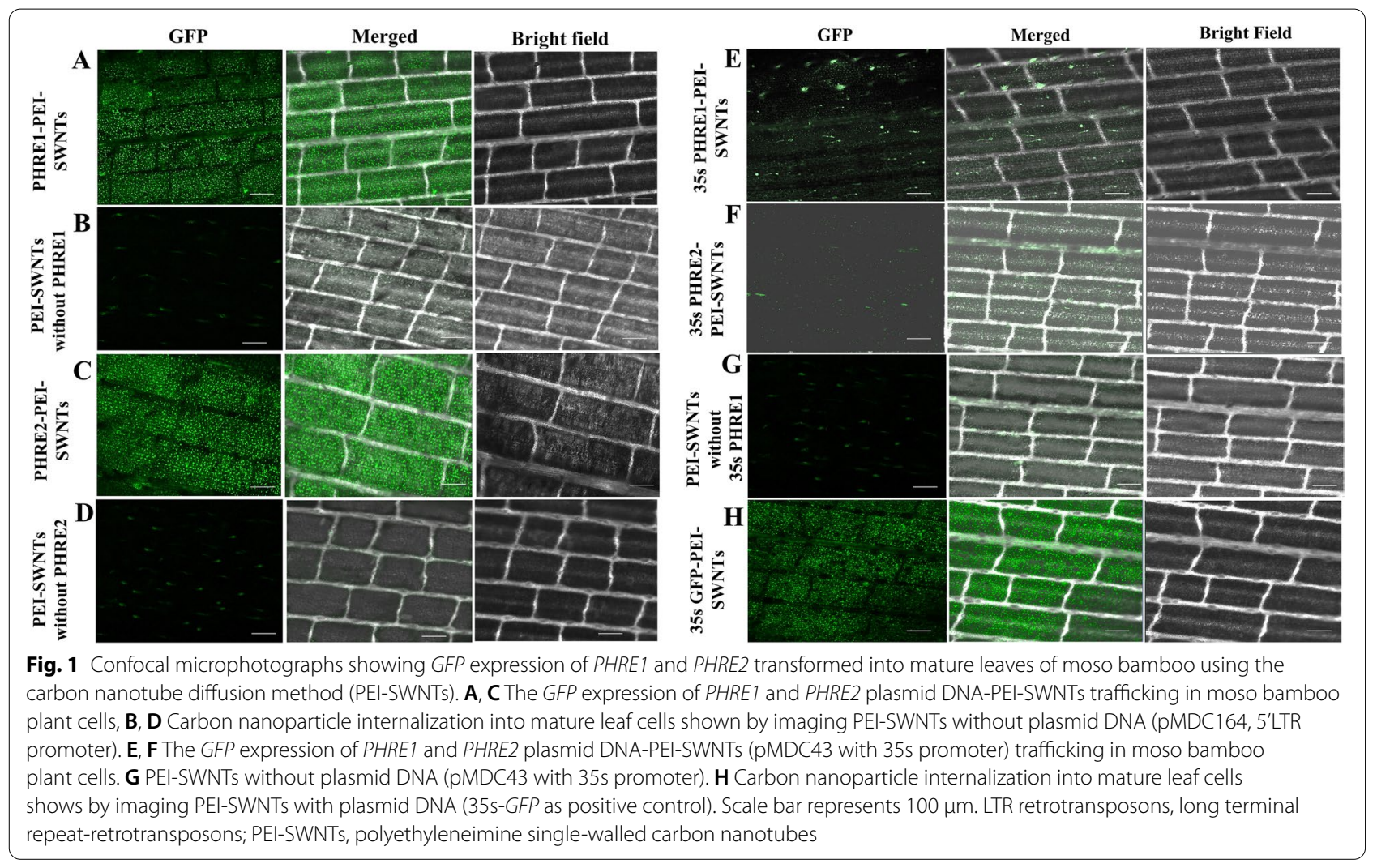

\section{PHRE1 and PHRE2 transcripts are expressed in roots and leaves}

The in-situ localization of PHRE1 and PHRE2 expression was identified in the root and leaves of bamboo. The probe hybridization using $306 \mathrm{bp}$ and $301 \mathrm{bp}$ fragments of 5'LTRs of PHRE1 and PHRE2, respectively, showed significant expression patterns in root cortex $(C)$, epidermis $(\mathrm{Ep})$, pericycle $(\mathrm{P})$, xylem $(\mathrm{X})$, and xylem parenchyma (XP) whereas in the leaves it was detected in endodermis and guard cells. Similarly, the same expression of PHRE2 was observed in the roots and leaves. Comparatively, PHRE1 displayed more diffused staining that appeared to be localized in the cells associated with root cortical cells (Fig. 4A) whereas PHRE2 expression was higher in the endodermis of leaf cells (Fig. 4D). These results were consistent with the RT-qPCR data. The hybridization signal was not detected in the roots and leaves using DIG-labeled sense probes of PHRE1 and PHRE2 (Fig. 4).

\section{PHRE1 and PHRE2 are activated by heat stress}

Based on the reporter genes expression, we performed additional molecular analysis to identify the precise function of both elements. Eleven Arabidopsis T1 lines were subjected to Southern blot assay to analyze the insertion polymorphism of PHRE1 and PHRE2 under heat stress. The DNA blots were probed with 5'LTR sequences of PHRE1 and PHRE2 separately, and the transposition of PHRE1 and PHRE2 was observed in heat-stressed progeny lines driven by 5' LTR promoter (Fig. 5A and B). PHRE1 allowed better discrimination of T1 lines (1 to 10) of Arabidopsis, compared to the control plant. Similarly, transposition copies of PHRE2 were observed in T1 lines 1 to 10 . We did not observe any transposition of PHRE1 and PHRE2 among the transgenes driven by the CaMV35s promoter in control plants (Fig. 5C and D). This indicated that not only the 5'LTR acted as a promoter for stable integration, but also could inherit the PHRE1 and PHRE2 elements into the progeny plants. By Southern hybridization, similar transposition activity of PHRE1 and PHRE2 was observed in moso bamboo wild type plants exposed to heat stress as well (Fig. 5E and F). However, we did not use bamboo transgenic plants developed using the carbon nanotube diffusion method, for copy number detection since plasmid vectors could not integrate into the genome $[49,50]$.

For identifying siRNAs expression of PHRE1 and PHRE2, Northern analysis in selected Arabidopsis T1 


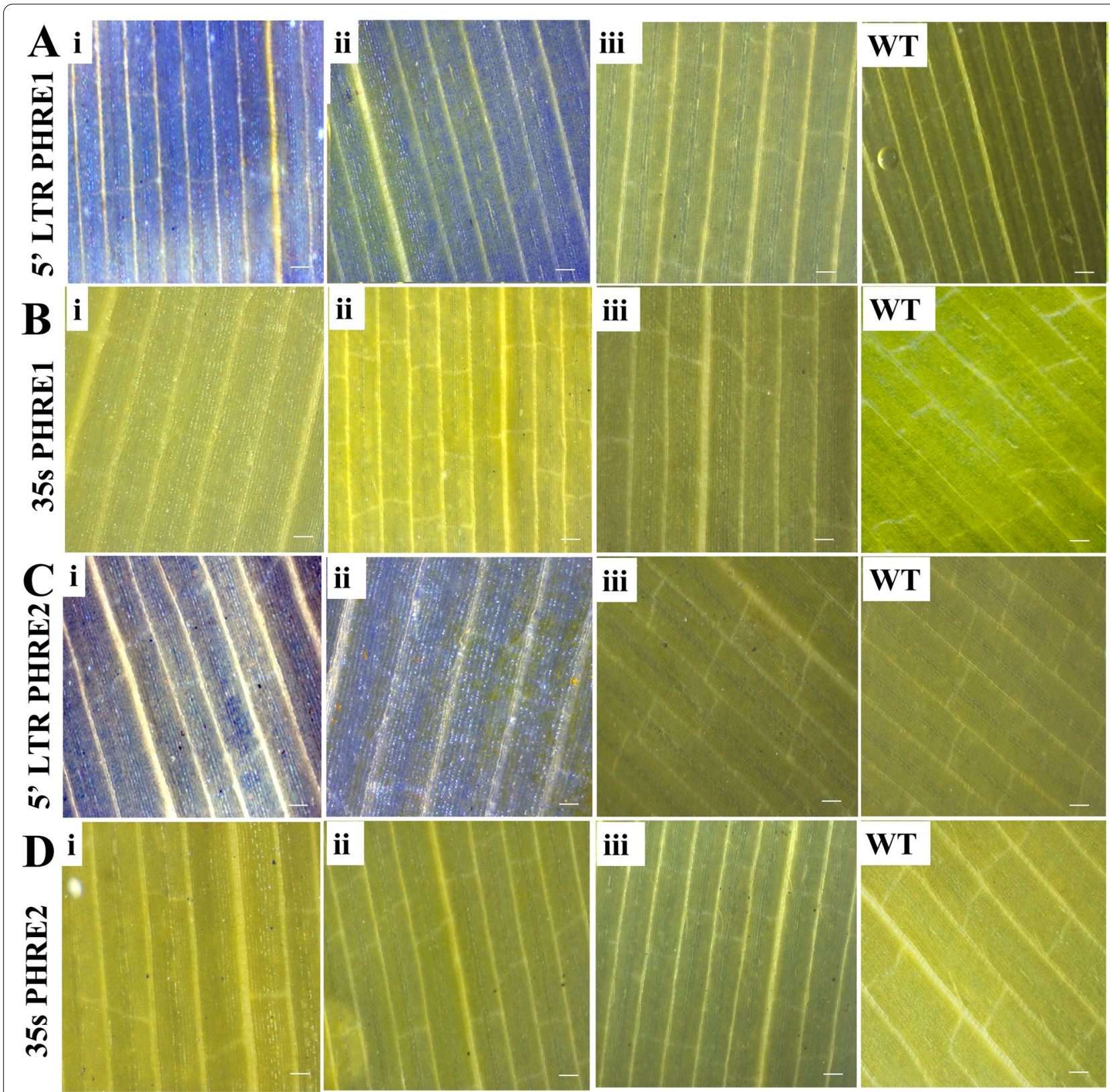

Fig. 2 GUS expression of PHRE1:pMDC164, PHRE2:pMDC164, PHRE1:pMDC43, and PHRE2:pMDC43 in transgenic moso bamboo leaves using carbon nanotubes diffusion transformation. Photographs showing the stained mature and young leaves infiltered by (A, C-i-ii) PHRE1 and PHRE2 (pMDC164) and (B, D-i-ii) PHRE1 and PHRE2 (pMDC43). A, B, C, D - iii, and WT are PEI-SWNTs without plasmid DNA, and (WT) plasmid DNA, respectively. Scale bar represents $50 \mu \mathrm{m}$

lines under heat stress resolved production of $22-24 \mathrm{bp}$ siRNAs specific to PHRE1 and PHRE2. The results were negative for the CaMV35s promoter-driven plants. The siRNAs isolated from wildtype control plants did not show any hybridization signal with their respective probes (Fig. 6). However, higher expressions of PHRE1 and PHRE2 were detected by qRT-PCR analysis in leaves of transgenic lines compared to control plants (Fig. S4).

\section{PHRE1 and PHRE2 retroelements show interactions} with TFs

Several TFs were predicted by scanning the JASPAR database such as TCP20, DOF2, DOF ZFP, DOF PBF, MYB1, WRKY40, MYB24, KANADI, WRKY18, MYB81, MYB119, GATA, DOF53, DOF57, MYB1, TCP4, TCP8, ethylene response 1, MYB113, NAC083, and MYB33, and their homologs were characterized from the moso bamboo genome database (Table S2). The qRT-PCR for 

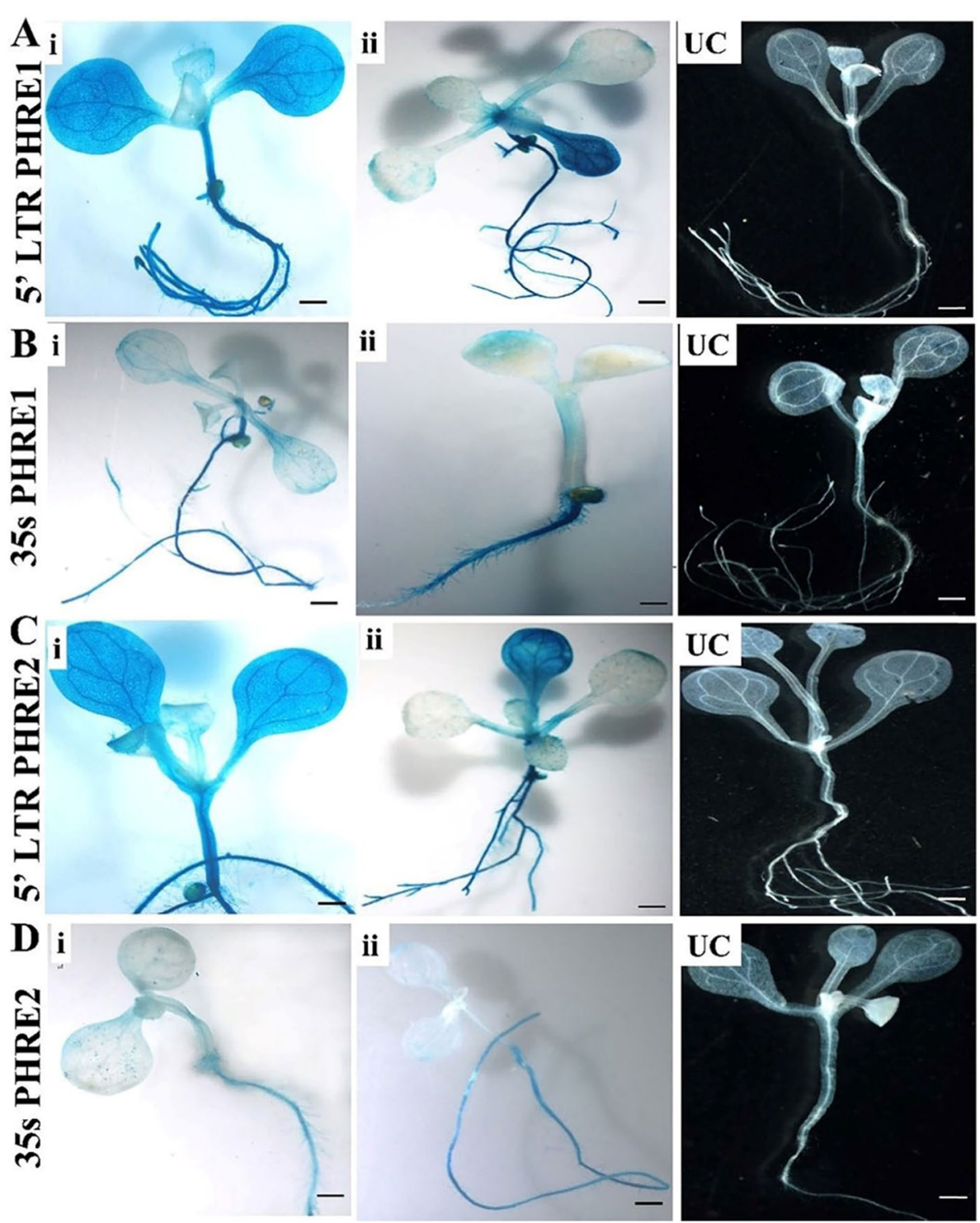

Fig. 3 Transgenic Arabidopsis plants showing GUS expression analysis of PHRE1 and PHRE2. A, C GUS expression analysis of pMDC164 construct of PHRE1 and PHRE 2 in transgenic Arabidopsis plants. Four weeks old T1 seedlings were incubated at $37^{\circ} \mathrm{C}$ for $24 \mathrm{~h}$. (i) $T 1$ transformants harboring pMDC164 construct with PHRE1 and PHRE2 (ii) transgenic plant under normal conditions and UC. B, D (i) GUS histochemical staining of T1 transformants harboring PMDC43 construct with PHRE1 and PHRE2. Four weeks old T1 seedlings were incubated at $37^{\circ} \mathrm{C}$ for $24 \mathrm{~h}$, (ii) transgenic plants without stress, and UC. The blue color indicates the level of expression and the PMDC164 construct with PHRE1 and PHRE2 showed stronger expression than the PMDC43 construct with PHRE1 and PHRE2. UC, untransformed control plant. Scale bar represents $20 \mu \mathrm{m}$

these TFs, resulted in only three showing significant differential expression in heat stressed plants than control plants. Assayed in root, leaf, and stem tissues collected under normal and heat stressed plants, statistically consistent and significant down-regulation of the target transcripts of three TFs, TCP20, DOF2, and GATA could be independently achieved in leaves and roots of plants subjected to heat-stress (Fig. S6). Our results suggested that TCP20, DOF2, and GATA are involved in the regulation of heat stress tolerance and host adaptation to environmental stress.

Since PHRE elements are also involved in heat stress response, our interest was to know whether any interactions existed between PHRE elements and TFs. Interestingly, in the yeast bait-prey assay, these TFs showed a specific pattern of interaction with PHRE elements. TCP20 (PH01001418G0330) and DOF2 (PH02Gene21543) were found to interact with PHRE1 

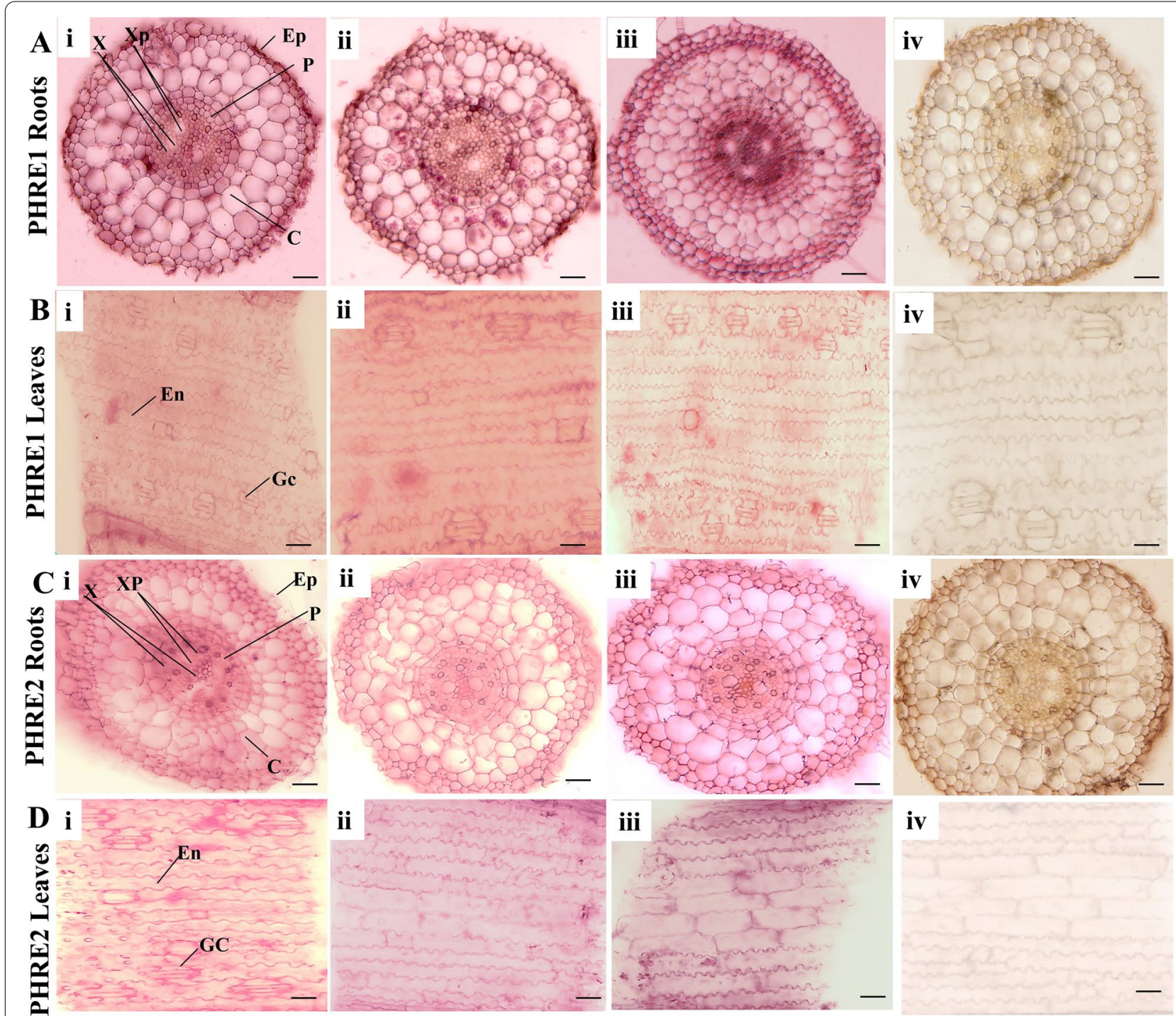

Fig. 4 In situ hybridization assay shows the expression patterns of PHRE1 and PHER2 in moso bamboo roots and leaves. Location of PHRE 1 expression signified by purple/blue color due to the enzymatic cleavage of a chromogenic substrate (5-Bromo-4-chloro-3-indolyl phosphate/nitro blue tetrazolium) by alkaline phosphatase-conjugated to anti-DIG antibody. (A, C-i-iii) In situ hybridization of cross-sections of primary and lateral roots, roots, and (B, D-i-iii) mature leaf blades with PHRE1 and PHRE2 antisense probes. (A, B, C, D-iv) negative control with sense probes. Roots and leaves were sampled from 30 -d-old seedlings treated under heat stress at $45^{\circ} \mathrm{C}$ for $4 \mathrm{~h}$. C, cortex; Ep, epidermis; P, pericycle; $X, x y l e m ; X P, x y l e m$ parenchyma; En, Endodermis and Gc, Guard cells. Scale bar represents $50 \mu \mathrm{m}$

in yeast cells, which were able to grow on the minimal medium containing SD/-Ura/-Trp/X-Gal (Fig. 7A). They did not seem to interact with the PHRE2 element. Whereas the GATA (PH02Gene06016) was the only TF that was found to interact with PHRE2, as the corresponding yeast cells could grow well on the minimal medium (Fig. 7B). The yeast transformants carrying negative control plasmids (AD, BD, AD-TCP20/DOF2/ GATA plus, BD, AD plus BD-PHRE1/PHRE2) were not able to grow (Fig.7). These results confirmed the selective interaction of TCP20 and DOF2 with PHRE1, and GATA with $P H R E 2$, further providing evidence for the promoter activity of 5'LTRs in PHRE1 and PHRE2 activation.

Based on the BiFC assay, strong GPF signals could be detected in the epidermal cells of pSPYNE-PHRE1: PSPYCE-TCP20, pSPYNE-PHRE1: pSPYCE-DOF2, and pSPYNE-PHRE2: pSPYCE-GATA (Fig. S7 and S8). No interactions were observed between the epidermal cells of tobacco leaves co-infiltered with negative controls. 


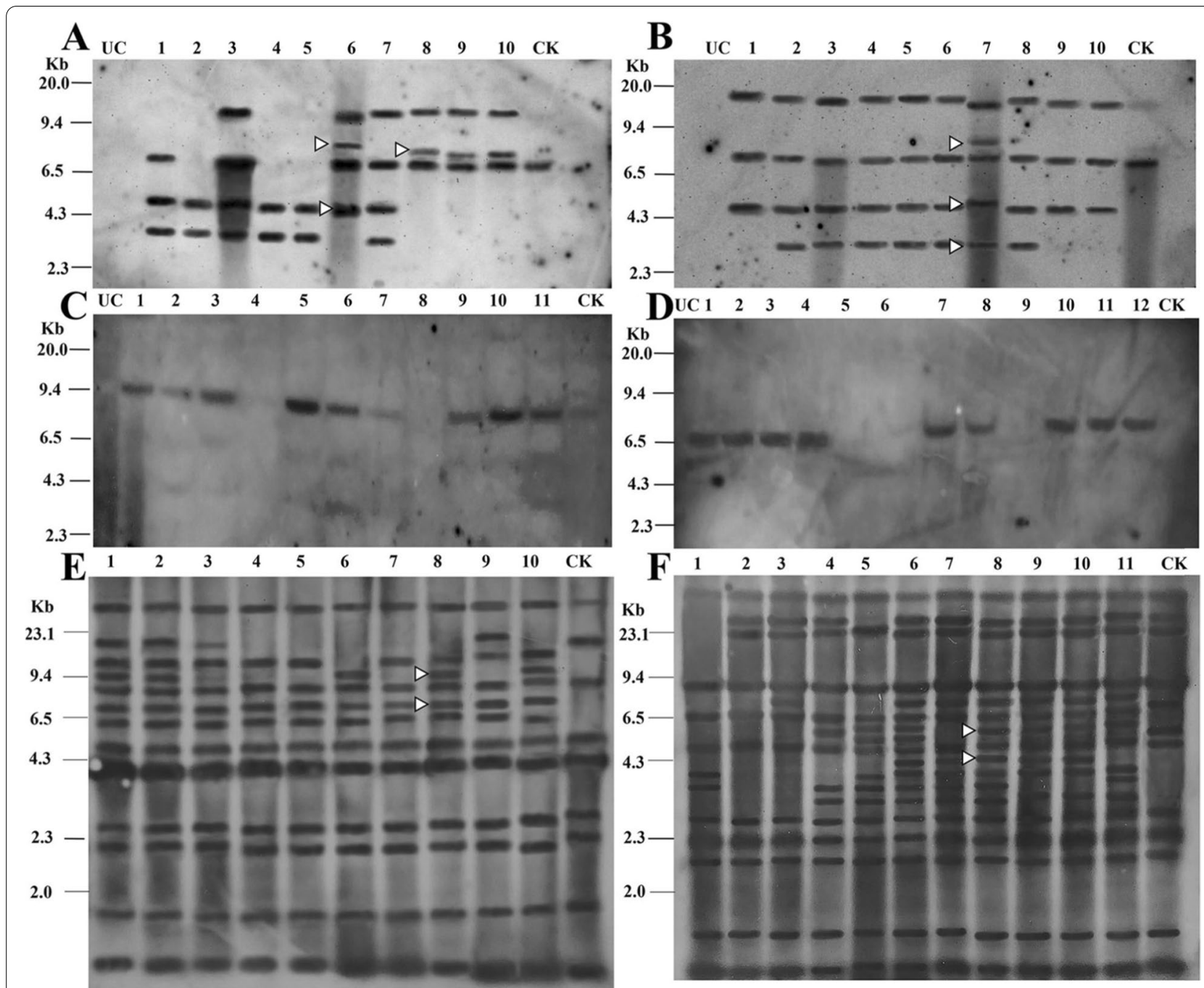

Fig. 5 Southern hybridization of Arabidopsis T1 lines harboring the pMDC164 and pMDC43 constructs and moso bamboo wild-type plants. Genomic DNA isolated from untransformed/empty vector control (UC) plants did not show any hybridization signal. Stressed plants and non-stressed control (CK) plants, digested with Pac1 or Hindlll enzyme showing hybridization signals, (A pMDC164, C pMDC43) PHRE1 and (B pMDC164, D pMDC43) PHRE2 5' LTR sequences were used as probes for hybridization of specific blots. Different lane numbers represent different transformant plants. Arrowheads indicate the transposed copies of LTRs. E and F, Southern hybridization for moso bamboo wild-type plants under heat stress. Genomic DNA isolated from stressed plants and non-stressed control (CK) plants, digested with Hindlll enzyme showing hybridization signals. (E) PHRE1 and (F) PHRE2 LTRs were used as probes for the hybridization of specific blots. Different lanes represent different plants

\section{Discussion}

The abundance of retroelements in the higher-order genome remains a biological mystery. Although they throw light on genome evolution, the elementary advantage of accumulating these elements remains unsolved. However, increasing evidence shows that these are involved in several homeostatic mechanisms including imparting stress tolerance [16, 26, 28, 46, 48]. Besides, they act epigenetically triggering transient gene expression associated with several biosynthetic pathways and regulatory elements. The biological role of the most common retroelement, the LTR retrotransposons, seems to be intricate and is majorly derived from its property of reverse transcription of their genomic RNA. Experimentally induced variants of LTR retrotransposon insertion confer stress responsiveness to nearby genes. Mobility bursts may occur which can generate novel, or alter stress-responsive regulatory gene networks [51, 52]. The stress-induced activation of LTR transposon has been shown to increase transgenerational transposition [53, 54]. The transcriptional activation of LTR retrotransposon is regulated by a siRNA-related pathway, 


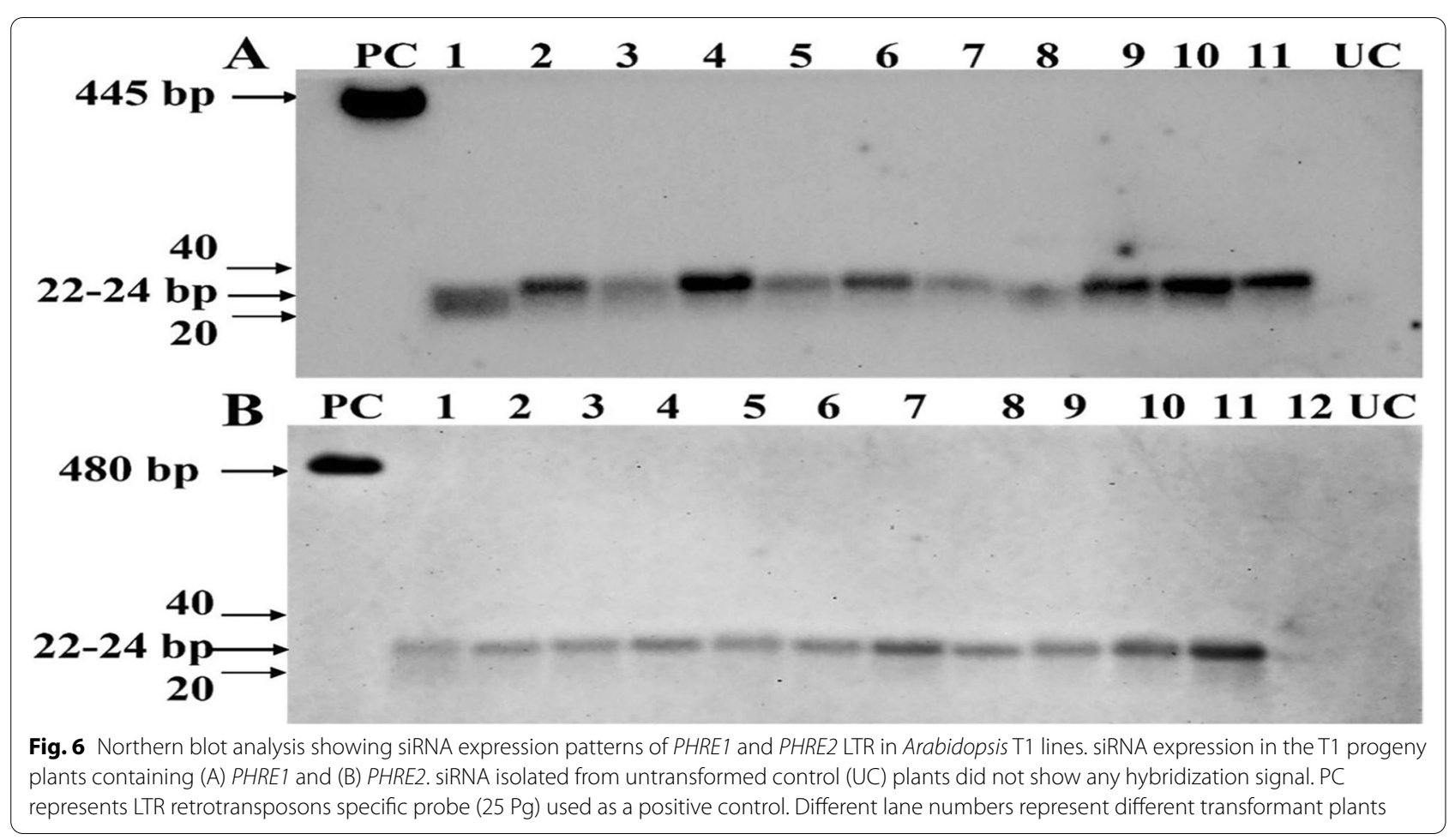

post-transcriptional modifications. These retroelements comprise trans-acting siRNAs, which are responsible for gene regulation at the transcriptional and post-transcriptional levels [20, 24, 55, 56]. LTR retrotransposons potentiate a unique balance by orchestrating tight stress regulation of physiological processes of plant growth and development. In deciphering the role of transposable elements, we make constant efforts to engineer/improve stress resistance in economically important crops. In this pursuit, we studied moso bamboo to decipher the role of two native LTR retrotransposons, PHRE1 and PHRE2, and their involvement in environmental stress, particularly heat stress.

We have used different biological systems to study the effect of PHRE1 and PHRE2 by estimating tissue-specific expression levels and their potential interactions with other genetic factors. As mentioned previously, moso bamboo is monocarpic and shows very infrequent sexual reproduction [57], due to its long vegetative growth and delayed flowering intervals. This pushes moso bamboo far behind in employing key bio-protocols that have been perfected in model systems such as Arabidopsis, rice, and tobacco. For instance, the development of transgenic plants by various genetic transformation techniques is technically difficult and extremely impractical in moso bamboo including micropropagation, in planta transformation by agroinfiltration, vacuum infiltration, floral dip, sonication, and gene delivery spraying [48]. Given this inconvenience, we have used SWNT transformation to deliver the LTR retrotransposons plasmid DNA into moso bamboo plants without transgene integration [50]. We could find that the internalization of nanoparticles in the transformant cells produced enhanced GFP expression levels in the leaves after $72 \mathrm{~h}$. Recently several reports have demonstrated carbon nanoparticles as efficient delivery systems of plant biomolecules such as DNA, RNA, and protein and are capable of strong internalization in planta [49]. The carbon nanotubes enable plasmid delivery without transgene integration into crop species [58-60], which are expressed across different tissues including leaves, roots protoplast, and immature tissues [59]. Enhanced GFP expression in leaf protoplasts through carbon nanotube delivery has been reported in wheat (Triticum aestivum) and arugula (Eruca sativa) [50]. Also, the use of nanoparticles mediated transformation has been demonstrated for siRNA production to silence genes [61, 62].

To demonstrate the in vivo expression pattern of PHRE1 and PHRE2 elements we have primarily used Arabidopsis, for transformation. The study revealed that the 5'LTRs of both the elements could show promoter activity in driving the expression of the GPF gene in Arabidopsis. The promoter activity of LTRs has been reported by several studies in different plant species $[52,63]$. These results are in agreement with previous reports of greater promoter activity of the 


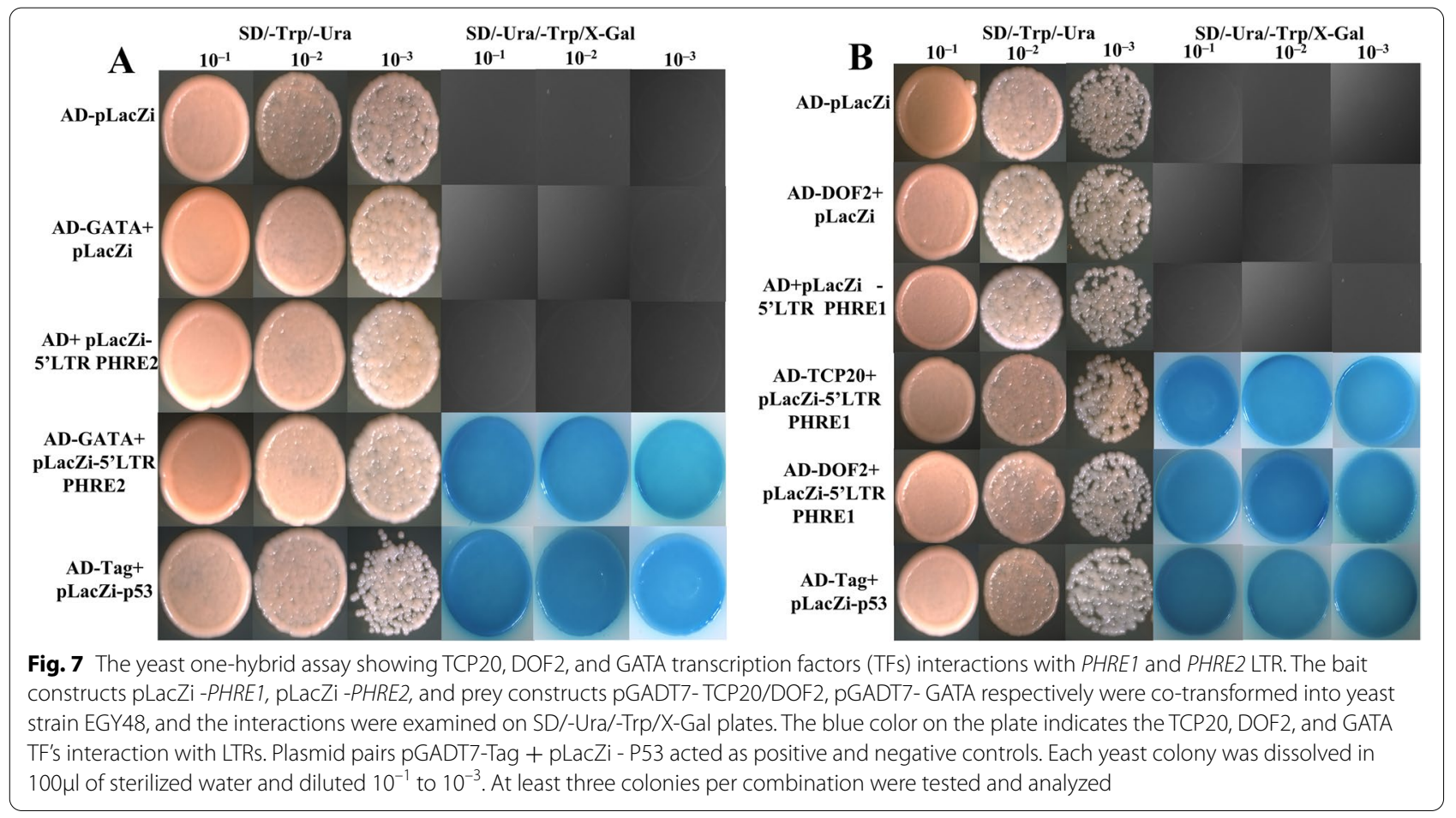

LTR in transgenic Arabidopsis [64, 65]. Takeda et al. (1999) demonstrated that tobacco Ttol promoter is responsible for enhanced expression patterns in transgenic tobacco lines by various stress. Remarkably, the specific role of 5 'LTR as a promoter could be observed in transcriptional activity under heat stress. Also, both the 5'LTRs promoters activated the transcription and transposition of PHRE1 and PHRE2 in the transgenic plants. When we overexpressed both PHRE1 and PHRE2 in moso bamboo using SWNTs transformation, a similar GPF expression could be noticed in the leaf tissues. Additionally, GUS activity also was found activated by the 5'LTR promoters in both Arabidopsis and moso bamboo. The promoter activity of 5'LTR was higher than CaMV35s, and no transposition activity could be detected driven by CaMV35s promoter. These results were subsequently proved by the quantitative gene expression studies, which showed a parallel pattern. Besides, elevated expression of 5'LTR, gag, pol, and 3'LTR of PHRE1 and PHRE2 indicated that the entire retroelements were activated in the heat-stressed plants than in the control plants. This suggested that heat stress could induce the increased transcriptional activity of LTR elements, and thereby increased transposition. Recent reports indicate the efficiency of LTR retrotransposons is achieved by copy number and transposition, under hormonal regulation and irradiation stress [48]. The moso bamboo seedlings generated by tissue culture expressing PHRE2 attained a significant increase in the copy number, suggesting its capability for retrotransposition [48]. Matsunaga et al. (2012) reported that Arabidopsis LTR retrotransposon ONSEN was inserted in the progeny of heat-stressed lines impaired in siRNAs demonstrating its role as heat stress promoter. Varying expression levels of LTR retrotransposons among different generations of transgenic lines, specifically the elevated expression 5' and 3' LTR in leaves, exemplified that PHRE1 and PHRE2 were stably integrated into the Arabidopsis genome.

We localized the expression of LTR retrotransposons in primary and lateral roots and matured leaves of moso bamboo. The enhanced expression in roots and leaves perhaps indicates the stress response role of these elements, because these organs are primary sensors of extraneous abiotic stresses, particularly heat, drought, salinity, etc. Tissue expression patterns indicated that PHRE1 was abundantly expressed in the cortex root cells but spread throughout the roots and leaves. PHRE2 was, however, predominantly expressed in roots associated with xylem and xylem parenchyma cells and in the guard cells of matured leaves. Our findings in moso bamboo draw parallels to the earlier reports of Gypsy retroelements in Brachiaria forage grasses [66]. Several LTR retrotransposons of the Ty3/Gypsy and Ty1/Copia family have been reported to 
predominantly be expressed in the sugarcane genome, suggesting that it has a specific crucial role in genetic variations, genome evolution, and adaptation to environmental stress [67].

We could detect abundant production of siRNA by these LTR retrotransposons, which was particularly high under heat stress. In biological systems, siRNAs are produced to regulate gene silencing and are involved predominantly in epigenetic processes [68]. The siRNA activity to regulate heat stress tolerance has already been reported in several systems such as Arabidopsis [26, 28, 69] mangrove [70], and Brassicaceae [27]. We also could observe the production of specific siRNAs in progeny lines. Heat stress significantly induced a higher accumulation of PHRE1-siRNA than PHRE2-siRNA. Transcriptional analysis indicated that moso bamboo LTR retrotransposons derived siRNAs (21-24 bp) might be involved in the transcriptional regulation of host genes [56]. In Arabidopsis, LTR retrotransposons in the chromatin gene $D D M 1$ are activated by mutations and produced 21-22-nt siRNAs involved in the regulation of epigenetic modification [71]. A recent study showed that LTR retrotransposons derived 24-nt siRNAs in Rhizophora apiculate could contribute to the progression of epigenetic TE silencing to maintain genetic diversity and thus an evolutionary response to stress conditions [70].

In addition, we confirmed LTR retrotransposon's interactions with different TFs (TCP20, DOF2, and GATA) confirming their intrinsic roles in plant growth regulation. Various TF gene families such as TCP [72], MYB [73], WRKY [74, 75], DOF (DNA-binding with one finger) [76] and GATA [77] have been analyzed in moso bamboo for their potential stress resistance functions. A recent analysis showed that heat shock TFs are involved in moso bamboo growth [78]. In our study, LTRs of PHRE1 interacted with two different TFs such as PhTCP20 and PhDOF2, while LTRs of PHRE2 interacted with $P h$ GATA, revealing its significant role in molecular function. The plant-specific TCP proteins contain a DNA binding domain (GGNCC), that plays a crucial role in the stress-associated regulation of plant growth and development [72, 79]. DOF proteins, comprising the DNA-binding C2C2-zinc domain, is the critical transcriptional regulator in plants for the different biological process including the regulation of plant growth and development in response to abiotic stress $[76,80]$. Similarly, the GATA proteins with a CX2CX18CX2C Zinc finger DNA-binding domain are involved in the regulation of plant hormone signal transduction and response to environmental stress [77, 81]. The quantitative analysis of overexpression of TFs in response to heat stress in moso bamboo leaf, root, and stem, revealed significant downregulation of PhTCP20 in the matured leaves, compared to the other two tissues. These results were consistent with a previous study that demonstrated the $P e$ TCP gene transcripts were significantly downregulated after the salicylic acid (SA) treatment in moso bamboo [72]. A similar downregulation, but in roots could be seen for PhDOF2 and PhGATA after exposure to heat. Wang et al. (2016) reported significant differential expression patterns of four $D O F$ genes associated with floral bud formation (PhDOF4, PhDOF5, PhDOF20 and $P h D O F 22$ ) under drought stress in moso bamboo plants, indicating positive regulation of the early stages of floral development. Downregulated expression patterns of PeGATA26 in moso bamboo and Arabidopsis seedlings were observed under the gibberellic acid treatment [82]. We could establish these interactions in our study, which was further validated through tobacco BiFC assay, which showed that PHRE1 and PHRE2 were localized in the guard cells of tobacco epidermal leaf, and interaction TFs had occurred at the protein level. However, the precise mechanisms of interaction remain to be solved. Several TFs are known to have their binding sites associated with LTRs, regulating cell specific gene expression [46]. In retroviral systems such as HIV1, specific interaction of regulatory elements to LTR domain in activating transposition machinery is reported [83]. In other viral systems, such as in the rice tungro virus, TF interactions with promotor regions are also known to occur [84]. Moreover, direct interactions of TFs with pol [85] and gag [86] have also been reported in HIV1. It would require additional explorations to know what precise regulatory mechanisms PHRE1 and PHRE2 undergo in moso bamboo. Barring the mode of interaction, our results and reported interactions suggest that transposon activity occurs unfettered in moso bamboo under stress such as heat, which drives regulation of developmental processes, and conferring abiotic stress tolerance as well.

Based on the results, we propose an activity flow for the PHRE elements in moso bamboo under heat stress (Fig. 8). On exposure to heat stress, the signals for the stress are activated in the leaves and roots. On stress sensing, the stress-dependent TFs, PhTCP, PhDOF2, and PhGATA bind to the 5'LTR of the PHRE elements and initiates the mRNA transcription. The entire retrotransposon sequence is transcribed and exported to the cytoplasm where the GAG and POL proteins produce virus like particles (VLPs). The reverse transcription follows to produce cDNAs of the complete retrotransposon which is imported back the nucleus to initiate transposition. By this activity, the copy number of the PHRE elements increases. Additionally, an epigenetic activity occurs when the mRNA forms a hairpin structure of double-stranded RNA, which is 


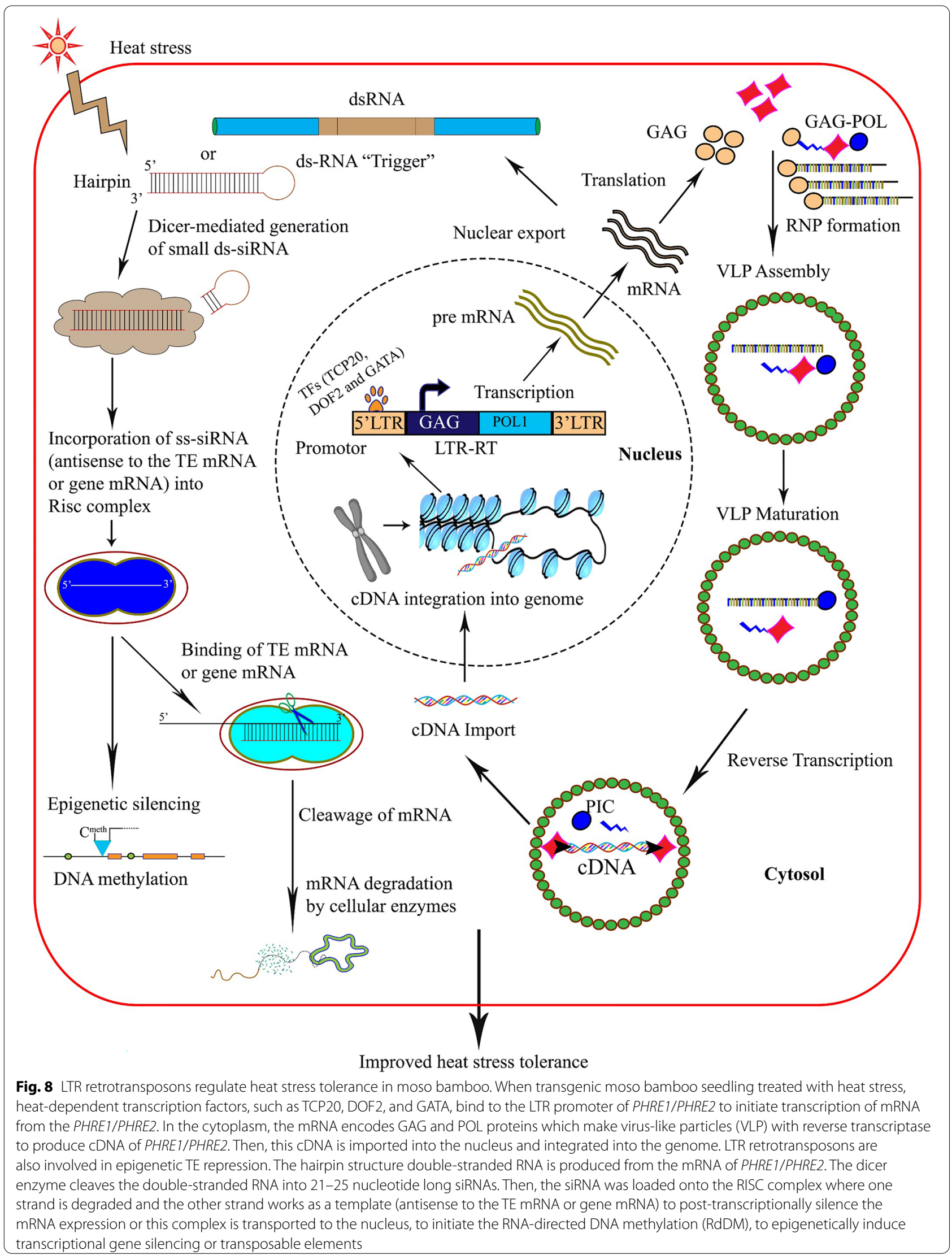


spliced by a dicer enzyme to 21-25 nucleotide long siRNAs. The siRNAs are subsequently loaded onto an RNA-induced silencing complex (RISC) where one of the strands gets degraded and the other acts as a template to silence mRNAs through pairing to the sense strand. Besides, RISC can also be imported to the nucleus to initiate RNA-directed DNA methylation (RdDM), which can induce specific silencing of the genes through epigenetic activity (Fig. 8). The specific silencing of genes could impart stress response in plants wherein certain metabolism is altered to offer increased protection to plant systems while conserving energy and resources.

\section{Conclusion}

Plant LTR retrotransposons are directly linked to genome evolution and integrity as well as connected to different stress responses, but their natural behavior remains unclear. In this study, we have explored the occurrence, functions, and interactions of two moso bamboo LTR retroelements, PHRE1 and PHRE2. Assaying using different biological systems, we could find that 5'LTR regions of both the elements show promoter activity and stress activation. A conspicuous transcription and transposition activity could be observed under heat stress. This could be related to their tissue specific expression patterns, as both are found in highly expressed roots and leaves, two major stress sensing organs in the plants. Under stress, the LTR element released several siRNAs indicating a role in epigenetic control. In addition, we could observe specific interactions with different TFs (PhTCP20, PhDOF2, and $P h$ GATA), which too was tissue specific. We could conclude that the PHRE1 and PHRE2 elements in moso bamboo, play several genetic roles such as promoter activity, transposition, tissue specific expression, epigenetic and gene-to-gene interactions while being activated significantly under stress. Also, the activity of these elements seemed largely dependent on adverse environmental factors. Taken together, the transcriptional activity of moso bamboo LTR retrotransposons provides a strong impetus for host adaptation to heat stress and their role as master regulators of the heat stress response.

\section{Material and Methods}

\section{Moso bamboo and Arabidopsis plant materials and growth} conditions

Moso bamboo seeds ( $P$. edulis) were collected from the host institute, Zhejiang Agriculture and Forestry University $\left(30^{\circ} 14^{\prime} \mathrm{N}, 119^{\circ} 42^{\prime} \mathrm{E}\right)$ in Lin'an, Zhejiang province, China. Seeds were germinated and plants were grown under greenhouse conditions. Fresh seeds picked from a single plant were surface sterilized with $70 \%$ ethanol for two minutes and washed with sterile distilled water. Subsequently, they were treated with $1 \%$ sodium hypochlorite $(\mathrm{NaClO})$ for eight minutes and washed with sterile distilled water to remove the sterilant. Later, the seeds were germinated in pots containing soilrite. The seedlings were maintained at $24-25^{\circ} \mathrm{C}$ for 30-45 days under a 16:8h light: dark photoperiod. To analyze the heat stress response, five-week-old seedlings were incubated in a programmed growth chamber at $45^{\circ} \mathrm{C}$ for four hours with $70 \% \mathrm{RH}$, and a $16: 8 \mathrm{~h}$ light: dark photoperiod. The seedlings grown under $24-25^{\circ} \mathrm{C}$ conditions were set as the control. After four hours, the plant samples were collected, flashed by freezing in liquid nitrogen and were stored at $-80^{\circ} \mathrm{C}$ for subsequent experiments.

Likewise, Arabidopsis seeds were surface sterilized in $70 \%$ ethanol for $10 \mathrm{~min}$ and washed with sterile water 5 times. The seeds were germinated in a petri dish containing half-strength Murashige and Skoog (MS) medium (Coolaber, China). The Petri dishes were incubated in dark at $4^{\circ} \mathrm{C}$ for 3 days and then transferred to a growth chamber. After 14 days, the healthy seedlings with well-established roots were transplanted into pots containing sterilized soil and soilrite mixture (2:1). The seedlings were watered on alternative days and maintained in a growth chamber at $24-25^{\circ} \mathrm{C}$ with $70 \% \mathrm{RH}$, and a 16:8h light: dark photoperiod. For heat stress treatment, a portion of the four-weeks old seedlings was exposed to $37^{\circ} \mathrm{C}$ for $24 \mathrm{~h}$ in another growth chamber with $70 \% \mathrm{RH}$, and a $16: 8 \mathrm{~h}$ light: dark photoperiod. The remaining unexposed seedlings were used as controls. After $24 \mathrm{~h}$, the plant tissues were collected, flashed by freezing in liquid nitrogen and were stored at $-80^{\circ} \mathrm{C}$.

\section{Isolation of PHRE1 and PHRE2}

Full-length target sequences of PHRE1 and PHRE2 were identified in the moso bamboo genome using LTRSTRUC software with default parameters as depicted in our previous work $[47,48]$. To clone PHRE1 and PHRE2, genomic DNA (500 ng) was extracted from fresh leaves using a plant genomic DNA kit (Tiangen, China) following the manufacturer's instructions. Later, PHRE1 (4.98 kb; Fig. S9A) and PHRE2 (5.51 kb; Fig. S9A), were amplified from the DNA using Phanta Max SuperFidelity DNA Polymerase enzyme (Vazyme, China) with sequence-specific primers (Table S3) as per the manufacturer's protocol. Subsequently, these fragments were cloned into the pUC18 vector, and the sequence insertion was ascertained by the Sanger sequencing method with different sets of primers (Table S4). 
PHRE1 and PHRE2 constructs for genetic transformation Full-length 5' LTR and open reading frame (ORF) sequences of PHRE1 with $\beta$-glucuronidase (GUS) reporter gene, and 3' LTR sequence of PHRE1 (5'LTR+gag + pol+GUS+3'LTR; a total of $8.3 \mathrm{~kb})$ were fused by overlap PCR. The final product was added between the attB1 (upstream) and attB2 (downstream) terminal ends by PCR. Primer details are given in Table S3. The attB-flanked PHRE1 was sub-cloned into an entry vector pDONR207, using BP clonase enzyme (Invitrogen). These fragments were subsequently cloned into two binary vectors, pMDC164 harboring no promoter, and pMDC43 harboring CaMV35s promoter, using recombination-based Gateway cloning technique, mediated by the LR clonase enzyme (Invitrogen). A similar cloning strategy was used for PHRE2 $\left(5^{\prime} \mathrm{LTR}+g a g+\right.$ pol + GUS +3'LTR; a total of $\left.9.3 \mathrm{~kb}\right)$ as mentioned above. The vector pMDC43 had a green fluorescent protein reporter gene (GFP) after the CaMV35s promoter, but the GFP was cloned between attB2 and hptII sites in the pMDC164 vector. Then, the recombinant clones were transformed into $E$. coli $\mathrm{DH} 5 \alpha$ competent cells. Target sequence orientation was confirmed by PCR assay using PHRE1 and PHRE2 specific forward and reverse primers (Table S3). To ensure the orientation of these inserts, only positive clones were sequenced by the sanger's method using different sets of primers.

\section{Transformation of PHRE1 and PHRE2 constructs into Arabidopsis plants}

Four different recombinant gateway constructs such as pMDC164:PHRE1, pMDC43:PHRE1, and pMDC164:PHRE2, and pMDC43:PHRE2 were individually transformed into Agrobacterium tumefaciens (LBA4404) competent cells by electroporation, and positive clones were selected using kanamycin according to a reported protocol $[87,88]$. Genetic transformation in Arabidopsis was achieved by floral dip method, wherein PHRE1 and PHRE2 were transformed into fully blossoming plants using Agrobacterium containing pMDC164 and pMDC43 vectors [89]. Transgenic plants were maintained in a growth chamber at the previous conditions, and after 30 days, transgenic seeds were collected and sterilized as described. The pMDC164 and pMDC43 have hygromycin phosphotransferase (hptII) gene as a selectable marker, and a minimum inhibitory concentration of $30 \mu \mathrm{g} / \mathrm{L}$ of hygromycin was used to screen the T1 plants. Seedlings that developed new shoots and survived in the minimum inhibitory concentration, were maintained in a growth chamber for two weeks and in a half-strength Hoagland medium. Transgenic plants with well-established roots and shoots were transferred to pots containing sterilized soilrite mixture for further experiments.
Arabidopsis plants transformed with Agrobacterium containing empty vector were used as control.

\section{Transformation of PHRE1 and PHRE2 constructs into moso bamboo}

We have used an efficient carbon nanotube (CNT) diffusion method [50] to transform moso bamboo seedlings with PHRE1 and PHRE2 constructs. Before infiltration to the leaf, PHRE1 and PHRE2 plasmid were mixed with polyethyleneimine (PEI)-single-walled carbon nanotubes (SWNT). Briefly, $30 \mathrm{mg}$ of dry carboxylate $(\mathrm{COOH})$ SWNTs (Sigma) was weighed, and bath sonicated for 10 $\mathrm{min}$ at room temperature, followed by continuous $30 \mathrm{~min}$ probe-tip sonication at $10 \%$ amplitude resulting in dark black solution in an ice bath. The absorbance of SWNTs was measured at $632 \mathrm{~nm}$ with an extinction coefficient of $0.36 \mathrm{~L} \mathrm{mg}^{-1} \mathrm{~cm}^{-1}$. 2-(N-morpholino) ethanesulfonic acid (MES) hydrate (Sigma-Aldrich) buffer solution at $\mathrm{pH} 5.5$ was added to react with $2 \mathrm{mg}$ of $\mathrm{COOH}-\mathrm{SWNTS}$ solution. Later, the carboxylic acid activators, ethyl carbodiimide (EDC, Sigma-Aldrich), and N-Hydroxysulfosuccinimide sodium salt (NHS, Sigma-Aldrich) was added dropwise to $\mathrm{COOH}-\mathrm{SWNTS}$ suspension and incubated at room temperature in a bath sonication for $15 \mathrm{~min}$. The suspension was transferred into prewashed Amicon 100k centrifugal filters (Merk) and centrifuged at $300 \mathrm{~g}$ for 8 min to remove the free EDC/NHS and biproducts. Activated $\mathrm{COOH}-\mathrm{SWNTS}$ reacted with a cationic polymer, polyethyleneimine (PEI), overnight on the orbital shaker at $180 \mathrm{rpm}$. The PEI-SWNTs suspension was transferred into $100 \mathrm{~K}$ centrifugal filters by centrifugation at $1000 \times \mathrm{g}$ for $20 \mathrm{~min}$. After repeated centrifugation, the absorbance of PEI-SWNTs was measured as described above. Typically, the $50 \mathrm{mg} / \mathrm{L}$ concentration of PEI-SWNTs suspension was adjusted for the mass ratio of 3:1 for PEISWNTs: plasmid DNA, by diluting with MES delivery buffer ( $25 \mathrm{mM} \mathrm{MES,} 15 \mathrm{mM} \mathrm{MgCl}_{2}$ at $\mathrm{pH}$ 6) per infiltration. Before infiltration, PEI-SWNTs buffer solution was incubated with targeted DNA (plasmid) at room temperature for $30 \mathrm{~min}$ to form the DNA-PEI-SWNTs complex. After the incubation, the DNA-PEI-SWNTs suspension was infiltered using a needleless syringe onto the abaxial surface of moso bamboo leaf. After $48 \mathrm{~h}$, a small portion of leaf tissue was cut and observed through the confocal microscope for GFP fluorescence to monitor the DNASWNTs efficiency.

\section{Locating the GUS reporter in the transgenic plants}

For identification of PHRE1 and PHRE2 functions under heat stress, the positively charged CNTs (PEI-SWNTs) were incubated with negatively charged plasmid DNA vectors, pMDC164 (without CaMV35s promoter), and pMDC43 (with CaMV35s promoter) containing PHRE1 
and PHRE2 and GUS as the reporter gene. Then, the plasmid vectors -PEI-SWNTs were infiltrated into moso bamboo leaves as described. To analyze the GUS expression in the T1 plants, a GUS histochemical assay was performed. Briefly, surface-sterilized seeds were germinated on a half-strength MS medium. A minimum inhibitory concentration of hygromycin $(30 \mu \mathrm{g} / \mathrm{L})$ was used to screen the T1 plants. Four-week-old, germinated seedlings were incubated in a programmed growth chamber at $37^{\circ} \mathrm{C}$ for $72 \mathrm{~h}$ with $70 \%$ relative humidity $(\mathrm{RH})$ and 16:8h light: dark photoperiod. Subsequently, seedlings were incubated overnight in GUS solution (Coolaber, China) at $37^{\circ} \mathrm{C}$, followed by four times washing with $70 \%$ ethanol and sterile water, respectively. The GUS expression blue spots in tissue were photographed under the microscope without damaging the tissue. Similarly, GUS histochemical assay was performed with five-week-old transgenic bamboo plants. Control was the seedlings transformed with Agrobacterium with empty vector, grown and processed as the samples, however, without hygromycin selection.

\section{Quantitative gene expression among the transgenics}

Genomic DNA was extracted from the harvested leaves of transgenic moso bamboo and Arabidopsis T1 lines using the cetyltrimethylammonium bromide (CTAB) method [90]. Putative transformants were confirmed by PCR assay using specific primer sets (5'LTR, GUS, 3'LTR, and hptII antibiotic marker) (Table S3). Amplicons were electrophoresed in $0.8 \%(\mathrm{w} / \mathrm{v})$ agarose gel. Positive transgenics were subjected to a quantitative real-time PCR (qRT-PCR) assay. For this, total RNA was extracted from the harvested root, leaf, and stem using an RNAiso Plus reagent (Takarabio) following the manufacturer's instructions. The reverse transcription into cDNA was carried out using a cDNA synthesis kit (Prime Script RT reagent Kit, Takara). The PCR assay was performed in a CFX96 Touch system (Bio-Rad) using TB Green Premix Ex Taq II (Takarabio). The $10 \mu \mathrm{l}$ reaction mix for each sample was contained $1.5 \mathrm{ng}$ of cDNA, $750 \mathrm{nM}$ of each forward and reverse primer with $5.5 \mu \mathrm{l}$ of TB Green premix. The amplification condition was set as a hot start of $95^{\circ} \mathrm{C}$ for $3 \mathrm{~min}$ followed by 40 cycles of $95^{\circ} \mathrm{C}$ for $15 \mathrm{~s}$ and $60^{\circ} \mathrm{C}$ for $1 \mathrm{~min}$. For determining the specificity of amplification, a melt curve analysis or dissociation program was run at $95^{\circ} \mathrm{C}$ for $15 \mathrm{~s} ; 60^{\circ} \mathrm{C}$ for $15 \mathrm{~s}$ followed by a slow ramp from 60 to $95^{\circ} \mathrm{C}$. Cycle threshold $(\mathrm{Ct})$ values of each sample were imported from CFX manager software version 2.3 (Bio-Rad, China). The reference genes used were actin for Arabidopsis and nucleotide tract-binding protein (NTB) for moso bamboo. Expression of 5'LTR, gag, pol and 3'LTR of PHRE1 and PHRE2 was quantified in different transgenic plants and was calculated as average
$\Delta \mathrm{Ct}$ values, i.e., the difference between the $\mathrm{Ct}$ means of transgene and reference genes. Three independent biological and three technical replicates were run. Primer details are enlisted in Table S3.

\section{Molecular characterization of transgenes}

Southern blotting was performed to analyze the transposition pattern of PHRE1 and PHRE2 in the PCR-positive transgenic and wild-type moso bamboo plants. Approximately $10 \mu \mathrm{g}$ of genomic DNA from each line was digested overnight at $37^{\circ} \mathrm{C}$ with $50 \mathrm{U}$ Pac1 or HindIII (New England Biolabs-High fidelity) for single cuts in the T-DNA. DNA digests were electrophoresed and resolved on $0.8 \%$ $(\mathrm{w} / \mathrm{v})$ agarose gel followed by transblotting to the positively charged nylon transfer membrane (Sigma-Aldrich, Amersham) by capillary action in $20 \times$ SSC buffer (3M $\mathrm{NaCl}, 0.3 \mathrm{M}$ sodium citrate, $\mathrm{pH}$ 7). The amplified products of 5'LTR of PHRE1 and PHRE2 generated from the respective pUC18 clones were used as a probe. Gel purified PCR products were labeled using a digoxigenin (DIG) probe synthesis kit (Sigma-Aldrich). Blot hybridization was carried out overnight in DIG Easy Hyb buffer solution at $42^{\circ} \mathrm{C}$ followed by washing twice with $0.5 \times$ saline sodium citrate (SSC) buffer at $65^{\circ} \mathrm{C}$. Probe corresponding to the coding region of PHRE1 and PHRE2 that hybridized with genomic DNA on the nylon membrane was detected by alkaline phosphatase-conjugated anti-DIG antibody followed by chemiluminescent substrate reaction (Sigma-Aldrich). Finally, the blots were exposed to $\mathrm{x}$-ray film (Fujifilm) and chemo doc imaging system (BioRad, China) for one hour at room temperature.

For the detection of siRNAs derived from PHRE1 and PHRE2 in transgenic plants, northern hybridization was carried out. The siRNAs were extracted from the fresh leaves of the transgenic plants using the Nucleospin miRNA isolation kit (Takarabio) following the manufacturer's instructions. Small RNAs $(10 \mu \mathrm{g})$ were denatured by heating at $68^{\circ} \mathrm{C}$ for $5 \mathrm{~min}$ and electrophoresed by resolving on $15 \%$ denaturing polyacrylamide gel followed by transblotting on a positively charged nylon transfer membrane (Sigma-Aldrich, Amersham). For probing, a 445 bp PCR product of the 5' LTR of PHRE1 and a $480 \mathrm{bp}$ of the 5' LTR of PHRE2 were used. Probes were labeled by PCR using a DIG probe synthesis kit (Sigma-Aldrich) as described previously [87]. Blot's hybridization was performed using DIG easy Hyb solution at $37^{\circ} \mathrm{C}$, followed by washing two times in $0.5 \times \mathrm{SSC}$ buffer at $42^{\circ} \mathrm{C}$. Chemiluminescence images were captured by using a chemo doc touch system (BioRad) according to the manufacturer's instructions.

\section{In situ localization of PHRE1 and PHRE2 transcripts}

In situ RNA hybridization was performed to localize transcripts of PHRE1 and PHRE2 in roots and leaf 
tissue of moso bamboo. The cDNA fragments of 5' LTR amplified from their respective pUC18 clones were used to synthesize DIG-labelled sense and antisense RNA probes by in vitro transcription. A $25 \mu \mathrm{l}$ reaction mix with DIG-labelled dUTP (Sigma-Aldrich) provided with gene-specific forward or reverse primers was used for transcription (Table S3). Bamboo tissue fixation, permeabilization, probe hybridization, and detection were performed according to previously described methods $[91,92]$. For root cross-section, roots were fixed in $4 \%$ paraformaldehyde at $4^{\circ} \mathrm{C}$ overnight, subsequently washed briefly with $0.1 \%$ phosphate buffered saline, and embedded in 5\% ultra-low gelling regular agarose (Takara, Clontech). The sections were cut into $100 \mu \mathrm{m}$ thickness using a Leica UC7 ultramicrotome (Leica Microsystems). Tissue specimens were mounted on glass microscope slides and examined in a Zeiss Imager M2m compound microscope.

\section{Analysis of transcription factors (TFs)}

Using LTR sequences of PHRE1 and PHRE2, TFs were predicted by querying the JASPAR 2020 database (http:// jaspar.genereg.net/). The homologous genes of TFs were characterized from the moso bamboo genome database (http://www.bamboogdb.org). Total RNA was isolated from the leaf, root, and stem of bamboo transgenic plants treated with heat stress as well as from the normally grown. The RNA was reverse transcribed into cDNA and the qRT-PCR was carried out. Fold change of the TFs expression was calculated by augmented comparative $\mathrm{Ct}$ method using $\log _{2}$ transformed mean data [93]. NTB of bamboo was used as the reference gene. Primer details are enlisted in Table S3.

\section{Cloning and confirmation of LTRs and TFs in bait and prey vectors}

Total RNA isolated from the fresh moso bamboo leaves using TRIzol Plus (Takara bio) was tested for quantity and quality using a Nanodrop ND-1000 spectrophotometer (Thermo Scientific). Approximately $400 \mathrm{ng}$ of purified RNA was reverse transcribed using a cDNA synthesis kit (Prime script RT, Takara). Fragments of TFs, TCP20, DOF2 (DNA binding with one finger), and GATA were amplified separately from the bamboo cDNA and cloned into a pESI-T vector (Yeasen, China) using specific primers. Primer details are given in Table S3. Five microliters of the ligated product were transformed into $100 \mu \mathrm{l}$ of DH5 $\alpha$ competent cells and selected on Luria broth (LB) plates with Ampicillin $(50 \mu \mathrm{g} / \mathrm{ml})$. Positive colonies were confirmed by PCR, using M13 primers. To confirm the successful cloning of the TFs inserts, colonies were sequenced using M13 primers by Sanger's method.
The 5' LTR fragments of PHRE1, PHRE2, and TFs (TCP20, DOF2, and GATA) were initially amplified from the pESI-T clone with $15 \mathrm{bp}$ overhangs at the upstream and downstream of the gene sequence, respectively, through PCR. Primer details are given in Table S3. To generate the bait construct, 5'LTR of PHRE1 and PHRE2 were individually cloned into Kpn1 and Xho1 sites of pLacZi, and the TFs (TCP20, DOF2, and GATA) were inserted downstream of Gal4 into pGADT7 as the prey construct (Fig. S9 B). Constructs were generated using the Clone press II One Step Cloning Kit (Vazyme, China). Five microliters of the ligated product were transformed into $100 \mu \mathrm{l}$ of DH5 $\alpha$ competent cells and selected on LB plates with Kanamycin $(50 \mu \mathrm{g} / \mathrm{ml})$. Positive colonies were confirmed by PCR using gene-specific primers (Table S3), followed by sequencing using CDS-specific primers. The sequence confirmed clone was transformed into yeast (Saccharomyces cerevisiae) competent strain EGY48 separately using high-efficiency polyethylene glycol (PEG)/LiAc-based method (Yeast transformation System2, Clontech, USA). Transformed yeast cells were selected on the minimal synthetic defined (SD) medium deficient in $\operatorname{Trp}(\mathrm{SD} /$-Trp) and Ura (SD/-Ura).

\section{Yeast one hybridization assay}

Yeast one-hybrid assay $(\mathrm{Y} 1 \mathrm{H})$ was performed by cotransformation of yeast strain EGY48 competent cells with plasmids. Activation domain (AD), pLacZi, ADTCP20+pLacZi, AD-DOF2+pLacZi, AD+5'LTR PHRE1, $\mathrm{AD}+5$ 'LTR $P H R E 2$ were selected as negative interaction, while AD-TCP20+pLacZi -5'LTR PHRE1, AD-DOF2+ pLacZi -5'LTR PHRE1, AD-GATA+pLacZi -5'LTR PHRE2, and AD-Tag/ pLacZi - P53 were selected as a positive interaction. The combinations were grown separately at $30^{\circ} \mathrm{C}$ in $50 \mathrm{ml} \mathrm{SD/-Trp} \mathrm{and} \mathrm{SD/-Leu,} \mathrm{shaken} \mathrm{at}$ $180 \mathrm{rpm}$ until OD600 reached $0.8(16-20 \mathrm{~h})$. Then cells were harvested by centrifugation at $1000 \times \mathrm{g}$ for $5 \mathrm{~min}$, and cell density was adjusted to $>1 \times 108$ cells per ml. One milliliter of each AD and pLacZi cell culture was added to $48 \mathrm{ml}$ of $2 \times$ yeast peptone dextrose adenine (YPDA) containing $50 \mu \mathrm{g} / \mathrm{ml}$ kanamycin and cultivated at $30^{\circ} \mathrm{C}$ at $50 \mathrm{rpm}$, examined under the microscope when zygotes appeared after 20-24 h. Cells were harvested by centrifugation at $1000 \times \mathrm{g}$ for $5 \mathrm{~min}$, the cell pellet was washed with $0.5 \times$ YPDA containing $50 \mu \mathrm{g} / \mathrm{ml}$ kanamycin and finally resuspended in $10 \mathrm{ml}$ of $0.5 \times$ YPDA containing $50 \mu \mathrm{g} / \mathrm{ml}$ kanamycin. To calculate the mating efficiency, $100 \mu \mathrm{l}$ of the mated culture $(1 / 10,1 / 100,1 / 1000$, and $1 / 10,000$ dilutions) was spread on SD/-Trp, SD/-Ura, and $\mathrm{SD} /$-Trp/-Ura agar plates and incubated at $30^{\circ} \mathrm{C}$ for $3-5$ days. The remaining culture was plated on $\mathrm{SD} /$-Trp/-Ura/ X-Gal $(40 \mu \mathrm{g} / \mathrm{ml} \mathrm{X-Gal)} \mathrm{agar} \mathrm{plates.} \mathrm{The} \mathrm{blue} \mathrm{colonies}$ that appeared on $\mathrm{SD} /$-Trp/-Ura/X-Gal agar plates were 
streaked on selection plates $\mathrm{SD} /-\mathrm{Trp} / \mathrm{Ura} / \mathrm{X}-\mathrm{Gal}$ and incubated at $30^{\circ} \mathrm{C}$ for $3-5$ days.

\section{Bimolecular fluorescence complementation assay} In-planta interactions of TFs (TCP20, DOF2, and GATA) with the 5'LTR promoters of PHRE1 and PHRE2 by using bimolecular complementation fluorescence (BiFC) assay in Nicotiana benthamiana (tobacco) plants and assessed the interactions using confocal microscopy. The fragments of PHRE1, PHRE2 (5'LTR $+g a g+p o l+3$ 'LTR) were separately inserted into the pSPYNE (Fig. S9 C) and the TFs into pSPYCE plasmids. Ligation and transformation were performed using the ClonExpress II One Step Cloning kit as explained above. All the constructs were confirmed by sequencing before transformation into A. tumefaciens strain GV3101. Different pairs of constructs were made such as PSPYNE-PHRE1: pSPYCE-TCP20, pSPYNE-PHRE1: pSPYCE-DOF2, and pSPYNE-PHRE2:pSPYCE-GATA as positive sets, and pSPYNE:pSPYCE, pSPYNE-PHRE1:pSPYCE, pSPYNE:pSPYCE-TCP20, pSPYNE:pSPYCE-DOF2, pSPYNE-PHRE2:pSPYCE, and pSPYNE:pSPYCE-GATA were used as negative controls. To test protein interactions, each pair of constructs was co-transformed into the abaxial side of four-week-old tobacco leaves. The GFP was examined in the nuclei of epidermal cells of transformed tobacco leaves carrying different constructs. Images of fluorescence and 4,6-diamidino-2-phenylindole (DAPI) staining of transfected plants were taken using a Zeiss LSM 510 Meta confocal laser scanning microscope after $48 \mathrm{~h}$ dark treatment, as previously described [94]. The plants transformed with $A$. tumefaciens harboring empty vector were treated as control. The primers used for the BiFC assay are listed in Table S3.

\section{Supplementary Information}

The online version contains supplementary material available at https://doi. org/10.1186/s12870-021-03339-1.

\section{Additional file 1.}

Additional file 2.

Additional file 3.

Additional file 4.

Additional file 5.

\section{Acknowledgments}

We would like to extend our sincere gratitude and appreciation to Professors Lin Xinchun and Jinbo Shen, Postdoctoral researcher Dr Naresh Vasupalli and Dr Jiang Cheng and their lab members, State Key Laboratory of Subtropical Silviculture, Zhejiang A\&F University, for their support of the experiments.

\section{Authors' contributions}

M.Z. conceived the project, designed the experiments, and provided overall supervision of the study, and commented on the manuscript. PKP performed the experiments. PKP and MR analyzed the data, interpreted the results, and wrote the manuscript. PKP, MR, KKV, LZ, KY, RK and MZ edited and revised the manuscript. All authors have read and approved the final manuscript.

\section{Funding}

This work was funded by a grant from the National Natural Science Foundation of China (Grant Nos 31870656, 31470615) and the Zhejiang Provincial Natural Science Foundation of China (Grant No. LZ19C160001).

\section{Availability of data and materials}

Supplementary Data for this article are available

\section{Declarations}

\section{Ethics approval and consent to participate}

The moso bamboo seeds used for this study were obtained from Zhejiang Agriculture and Forestry University, Hangzhou, China. The experimental research on plants performed in this study complies with institutional, national, and international guidelines.

\section{Consent for publication}

Not applicable.

\section{Competing interests}

All the authors have declared no conflict of interest.

\section{Author details}

${ }^{1}$ State Key Laboratory of Subtropical Silviculture, Zhejiang A\&F University, Lin'an, Hangzhou 311300, Zhejiang, China. ${ }^{2}$ Co-Innovation Center for Sustainable Forestry in Southern China, Nanjing Forestry University, Nanjing 210037, Jiangsu, China. ${ }^{3}$ Bamboo Research Institute, Nanjing Forestry University, Nanjing, 210037, Jiangsu, China. ${ }^{4}$ Division of Genetics, ICAR - Indian Agricultural Research Institute, New Delhi 110012, India. ${ }^{5}$ Helsinki Institute of Life Science HiLIFE, Biocenter 3, Viikinkaari 1, FI-00014 University of Helsinki, Helsinki, Finland. ${ }^{6}$ Zhejiang Provincial Collaborative Innovation Centre for Bamboo Resources and High-efficiency Utilization, Zhejiang A\&F University, Hangzhou 311300, Zhejiang, China.

Received: 9 September 2021 Accepted: 12 November 2021

Published online: 09 December 2021

References

1. Feschotte $C$, Jiang $N$, Wessler SR. Plant transposable elements: where genetics meets genomics. Nature Reviews Genetics. 2002;3(5):329-41.

2. Eickbush TH, Jamburuthugoda VK. The diversity of retrotransposons and the properties of their reverse transcriptases. Virus research. 2008;134(1-2):221-34.

3. Freed EO. HIV-1 gag proteins: diverse functions in the virus life cycle. Virology. 1998;251(1):1-15.

4. Bennetzen JL. Transposable element contributions to plant gene and genome evolution. Plant molecular biology. 2000;42(1):251-69.

5. Lisch D. How important are transposons for plant evolution? Nature Reviews Genetics. 2013;14(1):49-61.

6. Vitte C, Fustier M-A, Alix K, Tenaillon MI. The bright side of transposons in crop evolution. Briefings in Functional Genomics. 2014;13(4):276-95.

7. Negi P, Rai AN, Suprasanna P. Moving through the stressed genome: emerging regulatory roles for transposons in plant stress response. Frontiers in plant science. 2016;7:1448.

8. Bourque G, Burns KH, Gehring M, Gorbunova V, Seluanov A, Hammell M, et al. Ten things you should know about transposable elements. Genome biology. 2018;19(1):1-12.

9. Wessler SR. Plant retrotransposons: turned on by stress. Current Biology. 1996;6(8):959-61.

10. Pouteau S, Huttner E, Grandbastien MA, Caboche M. Specific expression of the tobacco Tnt1 retrotransposon in protoplasts. The EMBO journal. 1991;10(7):1911-8.

11. Takeda S, Sugimoto K, Otsuki H, Hirochika H. A 13-bp cis-regulatory element in the LTR promoter of the tobacco retrotransposon Tto 1 is 
involved in responsiveness to tissue culture, wounding, methyl jasmonate and fungal elicitors. The Plant Journal. 1999;18(4):383-93.

12. Lucas H, Feuerbach F, Kunert K, Grandbastien MA, Caboche M. RNA-mediated transposition of the tobacco retrotransposon Tnt1 in Arabidopsis thaliana. The EMBO Journal. 1995;14(10):2364-73.

13. Hirochika H, Otsuki H, Yoshikawa M, Otsuki Y, Sugimoto K, Takeda S. Autonomous transposition of the tobacco retrotransposon Tto1 in rice. The plant cell. 1996;8(4):725-34.

14. Kimura Y, Tosa Y, Shimada S, Sogo R, Kusaba M, Sunaga T, et al. OARE-1, a Ty1-copia retrotransposon in oat activated by abiotic and biotic stresses. Plant and cell Physiology. 2001;42(12):1345-54.

15. De Felice B, Wilson RR, Argenziano C, Kafantaris I, Conicella C. A transcriptionally active copia-like retroelement in Citrus limon. Cellular \& molecular biology letters. 2009;14(2):289-304.

16. Sun L, Jing Y, Liu X, Li Q, Xue Z, Cheng Z, Wang D, He H, Qian W. Heat stress-induced transposon activation correlates with 3D chromatin organization rearrangement in Arabidopsis. Nature communications. 2020;11(1):1-13.

17. Cao $Y$, Jiang $Y$, Ding $M$, He $S$, Zhang $H$, Lin $L$, et al. Molecular characterization of a transcriptionally active Ty1/copia-like retrotransposon in Gossypium. Plant cell reports. 2015;34(6):1037-47.

18. Nie Q, Qiao G, Peng L, Wen X. Transcriptional activation of long terminal repeat retrotransposon sequences in the genome of pitaya under abiotic stress. Plant Physiology and Biochemistry. 2019;135:460-8.

19. Ito H, Kim J-M, Matsunaga W, Saze H, Matsui A, Endo TA, et al. A stressactivated transposon in Arabidopsis induces transgenerational abscisic acid insensitivity. Scientific reports. 2016:6(1):1-12.

20. Marí-Ordóñez A, Marchais A, Etcheverry M, Martin A, Colot V, Voinnet O. Reconstructing de novo silencing of an active plant retrotransposon. Nature genetics. 2013;45(9):1029-39.

21. Mirouze M, Reinders J, Bucher E, Nishimura T, Schneeberger K, Ossowski $\mathrm{S}$, et al. Selective epigenetic control of retrotransposition in Arabidopsis. Nature. 2009:461(7262):427-30.

22. Burgess D, Li H, Zhao M, Kim SY, Lisch D. Silencing of mutator elements in maize involves distinct populations of small RNAs and distinct patterns of DNA Methylation. Genetics. 2020;215(2):379-91.

23. Cuerda-Gil D, Slotkin RK. Non-canonical RNA-directed DNA methylation. Nature plants. 2016;2(11):1-8.

24. Fultz D, Choudury SG, Slotkin RK. Silencing of active transposable elements in plants. Current opinion in plant biology. 2015;27:67-76.

25. Schorn AJ, Gutbrod MJ, LeBlanc C, Martienssen R: LTR-retrotransposon control by tRNA-derived small RNAs. Cell 2017, 170(1):61-71. e11.

26. Ito H, Gaubert H, Bucher E, Mirouze M, Vaillant I, Paszkowski J. An siRNA pathway prevents transgenerational retrotransposition in plants subjected to stress. Nature. 2011;472(7341):115-9.

27. Ito H, Yoshida T, Tsukahara S, Kawabe A. Evolution of the ONSEN retrotransposon family activated upon heat stress in Brassicaceae. Gene. 2013;518(2):256-61.

28. Matsunaga W, Ohama N, Tanabe N, Masuta Y, Masuda S, Mitani N, et al. A small RNA mediated regulation of a stress-activated retrotransposon and the tissue specific transposition during the reproductive period in Arabidopsis. Frontiers in Plant Science. 2015;6:48.

29. Cappucci U, Noro F, Casale AM, Fanti L, Berloco M, Alagia AA, et al. The Hsp70 chaperone is a major player in stress-induced transposable element activation. Proceedings of the National Academy of Sciences. 2019;116(36):17943-50

30. Kirov I, Omarov M, Merkulov P, Dudnikov M, Gvaramiya S, Kolganova E, Komakhin R, Karlov G, Soloviev A. Genomic and Transcriptomic Survey Provides New Insight into the Organization and Transposition Activity of Highly Expressed LTR Retrotransposons of Sunflower (Helianthus annuus L.). International journal of molecular sciences. 2020;21(23):9331.

31. Lanciano S, Cristofari G. Measuring and interpreting transposable element expression. Nature Reviews Genetics. 2020;21(12):721-36.

32. Finatto T, de Oliveira AC, Chaparro C, Da Maia LC, Farias DR, Woyann LG, et al. Abiotic stress and genome dynamics: specific genes and transposable elements response to iron excess in rice. Rice. 2015:8(1):1-18.

33. Gui Y, Wang S, Quan L, Zhou C, Long S, Zheng H, et al. Genome size and sequence composition of moso bamboo: a comparative study. Science in China Series C: Life Sciences. 2007;50(5):700-5.
34. Zhao H, Sun S, Ding Y, Wang Y, Yue $X$, Du X, et al. Analysis of 427 genomes reveals moso bamboo population structure and genetic basis of property traits. Nature Communications. 2021;12(1):1-12.

35. Tao G-Y, Ramakrishnan M, Vinod KK, Yrjälä K, Satheesh V, Cho J, et al. Multiomics analysis of cellular pathways involved in different rapid growth stages of moso bamboo. Tree physiology. 2020;40(11):1487-508.

36. Ramakrishnan M, Yrjälä K, Vinod KK, Sharma A, Cho J, Satheesh V, et al. Genetics and genomics of moso bamboo (Phyllostachys edulis): Current status, future challenges, and biotechnological opportunities toward a sustainable bamboo industry. Food and Energy Security. 2020;9(4):e229.

37. Clark L, Londoño X, Ruiz-Sanchez E. Bamboo taxonomy and habitat. In: Bamboo. Springer; 2015. p. 1-30.

38. Wei Q, Jiao C, Guo L, Ding Y, Cao J, Feng J, et al. Exploring key cellular processes and candidate genes regulating the primary thickening growth of M oso underground shoots. New Phytologist. 2017;214(1):81-96.

39. Peng Z, Lu Y, Li L, Zhao Q, Feng Q, Gao Z, et al. The draft genome of the fast-growing non-timber forest species moso bamboo (Phyllostachys heterocycla). Nature genetics. 2013;45(4):456-61.

40. Ramakrishnan M, Yrjälä K, Satheesh V, Zhou M-B: Bamboo Transposon Research: Current Status and Perspectives. Methods in Molecular Biology (Clifton, NJ) 2021, 2250:257-270.

41. Zhao H, Gao Z, Wang L, Wang J, Wang S, Fei B, Chen C, Shi C, Liu X, Zhang $\mathrm{H}$ : Chromosome-level reference genome and alternative splicing atlas of moso bamboo (Phyllostachys edulis). GigaScience. 2018;7(10):giy115.

42. Zhao H, Zhao S, Bamboo INf, Rattan, Fei B, Liu H, Yang H, Dai H, Wang D, Jin W et al: Announcing the Genome Atlas of Bamboo and Rattan (GABR) project: promoting research in evolution and in economically and ecologically beneficial plants. GigaScience 2017, 6(7):gix046.

43. Zhou M, Hu B, Zhu Y. Genome-wide characterization and evolution analysis of long terminal repeat retroelements in moso bamboo (Phyllostachys edulis). Tree Genetics \& Genomes. 2017;13(2):43.

44. Ramakrishnan M, Zhou M-B, Pan C-F, Hänninen H, Tang D-Q, Vinod KK. Nuclear export signal (NES) of transposases affects the transposition activity of mariner-like elements Ppmar1 and Ppmar2 of moso bamboo. Mobile DNA. 2019;10(1):1-12.

45. Ramakrishnan M, Zhou M, Pan C, Hänninen H, Yrjälä K, Vinod KK, et al. Affinities of terminal inverted repeats to DNA binding domain of transposase affect the transposition activity of bamboo Ppmar2 mariner-like element. International journal of molecular sciences. 2019;20(15):3692

46. Zhou S-S, Yan X-M, Zhang K-F, Liu H, Xu J, Nie S, et al. A comprehensive annotation dataset of intact LTR retrotransposons of 300 plant genomes. Scientific Data. 2021;8(1):1-9.

47. Zhou M, TANG D, ZHOU M. Cloning, characterization and phylogenetic analysis of a typical long terminal repeat retrotransposon in Phyllostachys heterocycla cv. pubescens. J Bamboo Res. 2014;33(3):1-10.

48. Zhou M, Liang L, Hänninen H. A transposition-active Phyllostachys edulis long terminal repeat (LTR) retrotransposon. Journal of plant research. 2018;131(2):203-10

49. Cunningham FJ, Goh NS, Demirer GS, Matos JL, Landry MP. Nanoparticlemediated delivery towards advancing plant genetic engineering. Trends in biotechnology. 2018;36(9):882-97.

50. Demirer GS, Zhang H, Matos JL, Goh NS, Cunningham FJ, Sung Y, et al. High aspect ratio nanomaterials enable delivery of functional genetic material without DNA integration in mature plants. Nature nanotechnology. 2019;14(5):456-64.

51. Grandbastien M-A: LTR retrotransposons, handy hitchhikers of plant regulation and stress response. Biochimica et Biophysica Acta (BBA)-Gene Regulatory Mechanisms 2015, 1849(4):403-416.

52. Galindo-González L, Mhiri C, Deyholos MK, Grandbastien M-A. LTRretrotransposons in plants: Engines of evolution. Gene. 2017;626:14-25.

53. Matsunaga W, Kobayashi A, Kato A, Ito H. The effects of heat induction and the siRNA biogenesis pathway on the transgenerational transposition of ONSEN, a copia-like retrotransposon in Arabidopsis thaliana. Plant and Cell Physiology. 2012;53(5):824-33.

54. Kalendar R, Sabot F, Rodriguez F, Karlov Gl, Natali L, Alix K: mobile elements and plant genome evolution, comparative analyzes and computational tools. In.; 2021. 
55. Li H, Freeling M, Lisch D. Epigenetic reprogramming during vegetative phase change in maize. Proceedings of the National Academy of Sciences. 2010;107(51):22184-9.

56. Zhou M, Zhu Y, Bai Y, Hänninen H, Meng X. Transcriptionally active LTR retroelement-related sequences and their relationship with small RNA in moso bamboo (Phyllostachys edulis). Molecular Breeding. 2017;37(10):1-11.

57. Gaubert H, Sanchez DH, Drost H-G, Paszkowski J. Developmental restriction of retrotransposition activated in Arabidopsis by environmental stress. Genetics. 2017;207(2):813-21.

58. Kwak S-Y, Lew TTS, Sweeney CJ, Koman VB, Wong MH, Bohmert-Tatarev K, et al. Chloroplast-selective gene delivery and expression in planta using chitosan-complexed single-walled carbon nanotube carriers. Nature nanotechnology. 2019;14(5):447-55.

59. Burlaka O, Pirko YV, Yemets A, Blume YB. Plant genetic transformation using carbon nanotubes for DNA delivery. Cytology and genetics. 2015;49(6):349-57.

60. Pantarotto D, Singh R, McCarthy D, Erhardt M, Briand JP, Prato M, et al. Functionalized carbon nanotubes for plasmid DNA gene delivery. Angewandte Chemie. 2004:116(39):5354-8.

61. Kam NWS, Dai H. Carbon nanotubes as intracellular protein transporters: generality and biological functionality. Journal of the American Chemical Society. 2005;127(16):6021-6.

62. Demirer GS, Zhang H, Goh NS, Pinals RL, Chang R, Landry MP: Carbon nanocarriers deliver siRNA to intact plant cells for efficient gene knockdown. Science advances 2020, 6(26):eaaz0495.

63. Kalendar R, Tanskanen J, Immonen S, Nevo E, Schulman AH. Genome evolution of wild barley (Hordeum spontaneum) by BARE-1 retrotransposon dynamics in response to sharp microclimatic divergence. Proceedings of the National Academy of Sciences. 2000;97(12):6603-7. https:// doi.org/10.1073/pnas.110587497

64. Grandbastien M-A. Activation of plant retrotransposons under stress conditions. Trends in plant science. 1998;3(5):181-7.

65. Pauls PK, Kunert K, Huttner E, Grandbastien M-A. Expression of the tobacco Tnt1 retrotransposon promoter in heterologous species. Plant molecular biology. 1994;26(1):393-402.

66. Santos FC, Guyot R, Do Valle CB, Chiari L, Techio VH, Heslop-Harrison P, et al. Chromosomal distribution and evolution of abundant retrotransposons in plants: gypsy elements in diploid and polyploid Brachiaria forage grasses. Chromosome research. 2015;23(3):571-82.

67. Mata-Sucre Y, Costa L, Gagnon E, Lewis GP, Leitch IJ, Souza G. Revisiting the cytomolecular evolution of the Caesalpinia group (Leguminosae): a broad sampling reveals new correlations between cytogenetic and environmental variables. Plant Systematics and Evolution. 2020;306(2):1-13.

68. Ito H. Small RNAs and transposon silencing in plants. Development, growth \& differentiation. 2012;54(1):100-7.

69. Masuta Y, Nozawa K, Takagi H, Yaegashi H, Tanaka K, Ito T, et al. Inducible transposition of a heat-activated retrotransposon in tissue culture. Plant and Cell Physiology. 2017;58(2):375-84.

70. Wang Y, Liang W, Tang T. Constant conflict between Gypsy LTR retrotransposons and $\mathrm{CHH}$ methylation within a stress-adapted mangrove genome. New Phytologist. 2018;220(3):922-35.

71. Lee SC, Ernst E, Berube B, Borges F, Parent J-S, Ledon P, et al. Arabidopsis retrotransposon virus-like particles and their regulation by epigenetically activated small RNA. Genome research. 2020;30(4):576-88.

72. Liu H-L, Wu M, Li F, Gao Y-M, Chen F, Xiang Y. TCP transcription factors in moso bamboo (Phyllostachys edulis): genome-wide identification and expression analysis. Frontiers in plant science. 2018;9:1263.

73. Yang K, Li Y, Wang S, Xu X, Sun H, Zhao H, et al. Genome-wide identification and expression analysis of the MYB transcription factor in moso bamboo (Phyllostachys edulis). PeerJ. 2019;6:e6242.

74. Huang R, Gao H, Liu J, Li X. WRKY transcription factors in moso bamboo that are responsive to abiotic stresses. Journal of Plant Biochemistry and Biotechnology. 2021:1-8.

75. Wu M, Liu H, Han G, Cai R, Pan F, Xiang Y. A moso bamboo WRKY gene PeWRKY83 confers salinity tolerance in transgenic Arabidopsis plants. Scientific reports. 2017;7(1):1-16.

76. Cheng Z, Hou D, Liu J, Li X, Xie L, Ma Y, et al. Characterization of moso bamboo (Phyllostachys edulis) Dof transcription factors in floral development and abiotic stress responses. Genome. 2018;61(3):151-6.
77. Wang T, Yang Y, Lou S, Wei W, Zhao Z, Ren Y, et al. Genome-wide characterization and gene expression analyses of GATA transcription factors in Moso bamboo (Phyllostachys edulis). International journal of molecular sciences. 2020;21(1):14.

78. Xie L, Li X, Hou D, Cheng Z, Liu J, Li J, et al. Genome-wide analysis and expression profiling of the heat shock factor gene family in Phyllostachys edulis during development and in response to abiotic stresses. Forests. 2019;10(2):100

79. Yang Z, Zhu P, Kang H, Liu L, Cao Q, Sun J, et al. High-throughput deep sequencing reveals the important role that microRNAs play in the salt response in sweet potato (Ipomoea batatas L.). BMC genomics. 2020:21(1):1-16.

80. Wang T, Yue J-J, Wang X-J, Xu L, Li L-B, Gu X-P. Genome-wide identification and characterization of the Dof gene family in moso bamboo (Phyllostachys heterocycla var. pubescens). Genes \& Genomics. 2016;38(8):733-45.

81. An Y, Zhou Y, Han X, Shen C, Wang S, Liu C, et al. The GATA transcription factor GNC plays an important role in photosynthesis and growth in poplar. Journal of experimental botany. 2020;71(6):1969-84.

82. Wang T, Yang Y, Lou S, Wei W, Zhao Z, Lin C, Ma L. Genome-wide analysis of GATA factors in moso bamboo (Phyllostachys edulis) unveils that PeGATAs regulate shoot rapid-growth and rhizome development. bioRxiv 2019:744003.

83. Karn J, Stoltzfus CM. Transcriptional and posttranscriptional regulation of HIV-1 gene expression. Cold Spring Harbor perspectives in medicine. 2012;2(2):a006916.

84. Petruccelli S, Dai S, Carcamo R, Yin Y, Chen S, Beachy RN. Transcription factor RF2a alters expression of the rice tungro bacilliform virus promoter in transgenic tobacco plants. Proceedings of the National Academy of Sciences. 2001:98(13):7635-40.

85. Goffin V, Demonte D, Vanhulle C, De Walque S, De Launoit Y, Burny A, et al. Transcription factor binding sites in the pol gene intragenic regulatory region of HIV-1 are important for virus infectivity. Nucleic acids research. 2005;33(13):4285-310.

86. Miyakawa K, Nishi M, Matsunaga S, Okayama A, Anraku M, Kudoh A, et al. The tumour suppressor APC promotes HIV-1 assembly via interaction with Gag precursor protein. Nature communications. 2017;8(1):1-13.

87. Papolu PK, Gantasala NP, Kamaraju D, Banakar P, Sreevathsa R, Rao U. Utility of host delivered RNAi of two FMRF amide like peptides, flp-14 and flp-18, for the management of root knot nematode. Meloidogyne incognita. PLoS One. 2013:8(11):e80603.

88. Dutta TK, Papolu PK, Banakar P, Choudhary D, Sirohi A, Rao U. Tomato transgenic plants expressing hairpin construct of a nematode protease gene conferred enhanced resistance to root-knot nematodes. Frontiers in microbiology. 2015;6:260

89. Clough SJ, Bent AF. Floral dip: a simplified method for Agrobacteriummediated transformation of Arabidopsis thaliana. The plant journal. 1998;16(6):735-43.

90. Murray M, Thompson WF. Rapid isolation of high molecular weight plant DNA. Nucleic acids research. 1980;8(19):4321-6.

91. Kimber MJ, Fleming CC, Prior A, Jones JT, Halton DW, Maule AG. Localisation of Globodera pallida FMRFamide-related peptide encoding genes using in situ hybridisation. International journal for parasitology. 2002;32(9):1095-105.

92. Athman A, Tanz SK, Conn VM, Jordans C, Mayo GM, Ng WW, et al. Protocol: a fast and simple in situ PCR method for localising gene expression in plant tissue. Plant methods. 2014;10(1):1-20.

93. Dutta TK, Papolu PK, Singh D, Sreevathsa R, Rao U. Expression interference of a number of Heterodera avenae conserved genes perturbs nematode parasitic success in Triticum aestivum. Plant Science. 2020;301:1 10670.

94. Gnanasekaran P, Ponnusamy K, Chakraborty S. A geminivirus betasatellite encoded $\beta C 1$ protein interacts with PsbP and subverts PsbP-mediated antiviral defence in plants. Molecular plant pathology. 2019;20(7):943-60.

\section{Publisher's Note}

Springer Nature remains neutral with regard to jurisdictional claims in published maps and institutional affiliations. 